\title{
Evolutionary enhancement of Zika virus infectivity in Aedes aegypti mosquitoes
}

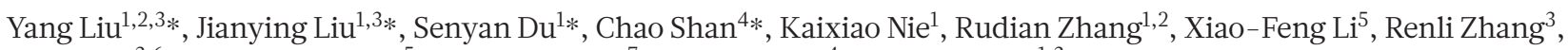
Tao Wang ${ }^{3,6}$, Cheng-Feng Qin ${ }^{5}$, Penghua Wang ${ }^{7}$, Pei-Yong Shi ${ }^{4}$ \& Gong Cheng ${ }^{1,3}$

Zika virus (ZIKV) remained obscure until the recent explosive outbreaks in French Polynesia (2013-2014) and South America (2015-2016) ${ }^{1-3}$. Phylogenetic studies have shown that ZIKV has evolved into African and Asian lineages. The Asian lineage of ZIKV was responsible for the recent epidemics in the Americas ${ }^{1,3}$. However, the underlying mechanisms through which ZIKV rapidly and explosively spread from Asia to the Americas are unclear. Non-structural protein 1 (NS1) facilitates flavivirus acquisition by mosquitoes from an infected mammalian host and subsequently enhances viral prevalence in mosquitoes ${ }^{4}$. Here we show that NS1 antigenaemia determines ZIKV infectivity in its mosquito vector Aedes aegypti, which acquires ZIKV via a blood meal. Clinical isolates from the most recent outbreak in the Americas were much more infectious in mosquitoes than the FSS13025 strain, which was isolated in Cambodia in 2010. Further analyses showed that these epidemic strains have higher NS1 antigenaemia than the FSS13025 strain because of an alanine-to-valine amino acid substitution at residue 188 in NS1. ZIKV infectivity was enhanced by this amino acid substitution in the ZIKV FSS13025 strain in mosquitoes that acquired ZIKV from a viraemic C57BL/6 mouse deficient in type I and II interferon (IFN) receptors (AG6 mouse). Our results reveal that ZIKV evolved to acquire a spontaneous mutation in its NS1 protein, resulting in increased NS1 antigenaemia. Enhancement of NS1 antigenaemia in infected hosts promotes ZIKV infectivity and prevalence in mosquitoes, which could have facilitated transmission during recent $Z I K V$ epidemics.

Zika virus (ZIKV), a mosquito-borne virus belonging to the genus flavivirus of the family Flaviviridae, is transmitted to humans by Aedes mosquito species ${ }^{1,5}$. Several neurological complications, such as Guillain-Barré syndrome in adults ${ }^{6}$ and microcephaly in newborn babies $^{7}$, have been associated with ZIKV infection. Before 2007, cases of ZIKV infection were detected only sporadically ${ }^{1,8}$. The first reported mild ZIKV outbreak occurred in 2007 on Yap Island in Western Pacific Micronesia ${ }^{9}$. In 2013, a larger ZIKV epidemic was recorded in French Polynesia and other Southern Pacific islands ${ }^{2,10}$. In 2015, ZIKV emerged in the Americas for the first time, rapidly spreading to 20 countries or territories ${ }^{3}$. Phylogenetic studies have revealed that ZIKV has evolved into African and Asian clusters ${ }^{1,3}$. Sequence analyses have shown that the ZIKV strains that caused the earlier outbreaks in Micronesia (2007) and French Polynesia (2013-2014) and later outbreaks in the Americas (2015-2016) all belong to the Asian clade ${ }^{1}$, suggesting that increased infectivity of the Asian ZIKV lineage might have contributed to the recent epidemic.

Evolutionary increases in the infectivity of mosquito-borne viruses within their vectors results in high epidemic potential ${ }^{11,12}$. We speculate that the adaptability and infectivity of ZIKV within its mosquito vectors

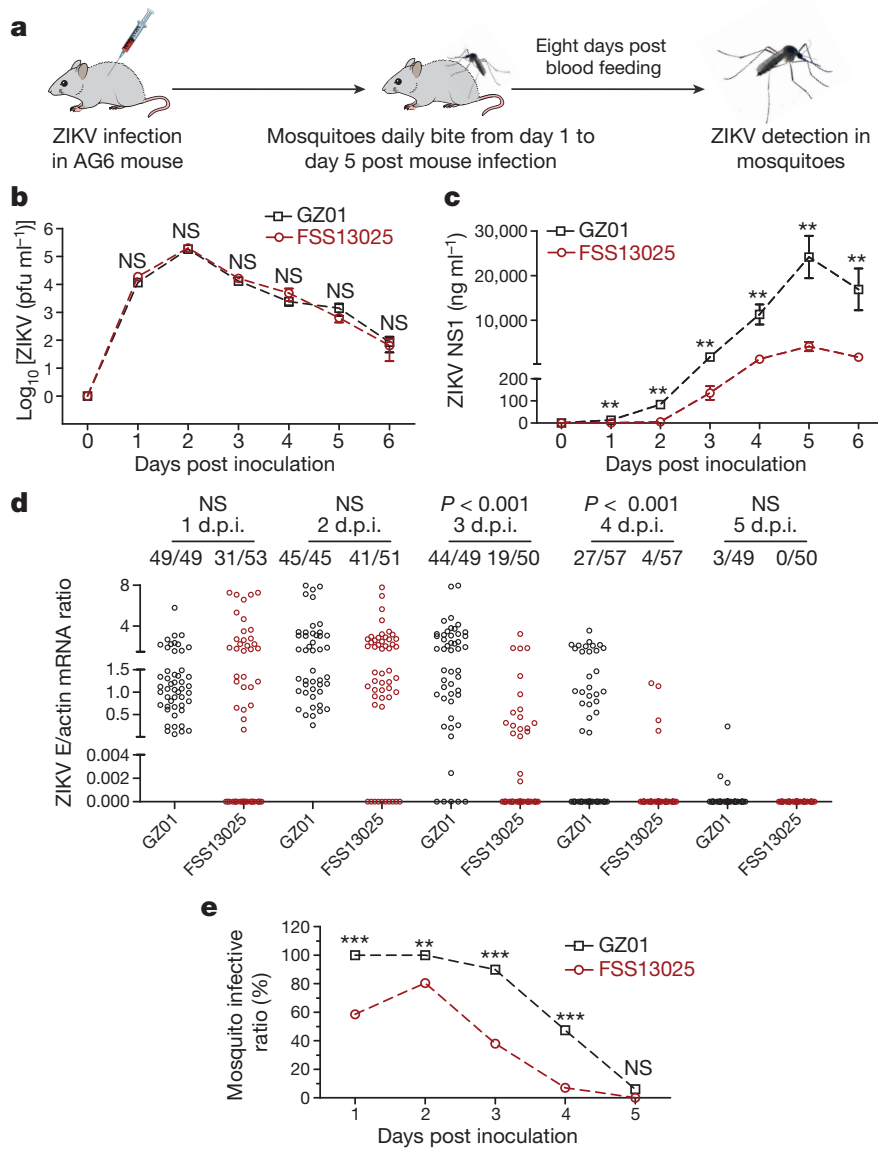

Figure 1 Comparing the infectivity of ZIKV isolates of Asian lineage in mosquitoes. a, Schematic representation of the study design. Separated groups of AG6 mice were intradermally infected with $1 \times 10^{4}$ p.f.u. of the GZ01 and FSS13025 strains. b, Detection of the ZIKV load in the blood plasma by plaque assay ( $n=14$ mice per group pooled from 5 independent biological replicates). c, ZIKV NS1 measurement by enzyme-linked immunosorbent assay (ELISA; $n=6$ mice per group pooled from 3 independent biological replicates). d, e, Comparison of the infectivity of two ZIKV isolates in A. aegypti ( $n=6$ mice per group pooled from 3 independent biological replicates). The number at the top of each column represents infected number/total number. Each dot represents a mosquito (d). The data are represented as the percentage of mosquito infections (e). d.p.i., days post inoculation. Data are mean \pm s.e.m. (b, c). $P$ values were determined by two-tailed Mann-Whitney test $(\mathbf{b}-\mathbf{d})$ or twosided Fisher's exact test (e). ${ }^{* *} P<0.01, * * * P<0.001$, NS, not significant.

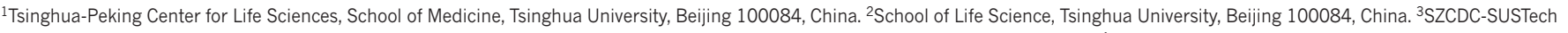
Joint Key Laboratory for Tropical Diseases, Shenzhen Center for Disease Control and Prevention, Shenzhen, Guangdong 518055, China. ${ }^{4}$ Department of Biochemistry \& Molecular Biology, Department of Pharmacology \& Toxicology, and Sealy Center for Structural Biology \& Molecular Biophysics, University of Texas Medical Branch, Galveston, Texas 77555, USA. ${ }^{5}$ State Key Laboratory of Pathogen and Biosecurity, Beijing Institute of Microbiology and Epidemiology, Beijing 100071, China. ${ }^{6}$ Department of Biology, Southern University of Science and Technology, Nanshan, Shenzhen, Guangdong 518055, China. ${ }^{7}$ Department of Microbiology and Immunology, School of Medicine, New York Medical College, Valhalla, New York 10595 , USA.

*These authors contributed equally to this work.
} 


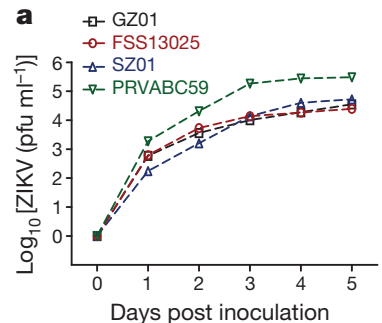

d

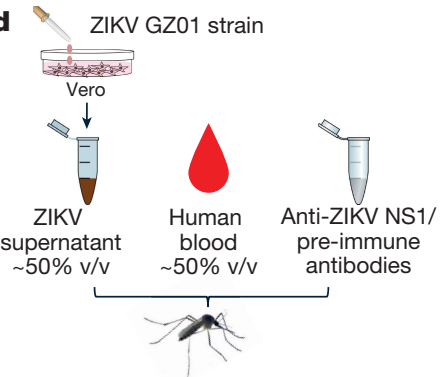

9

g ZIKV FSS13025 strain
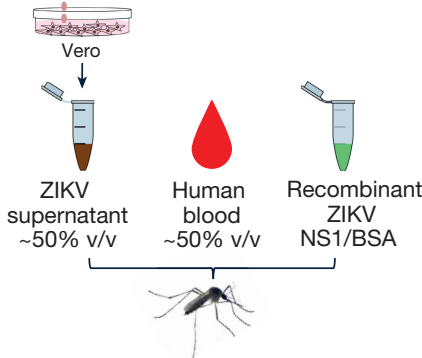

j

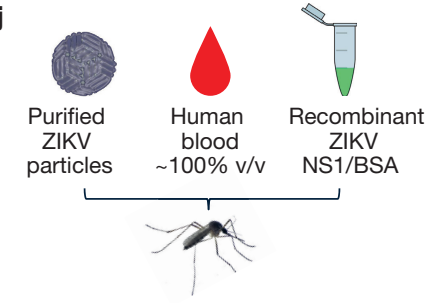

b

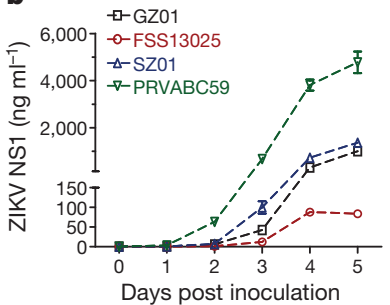

e

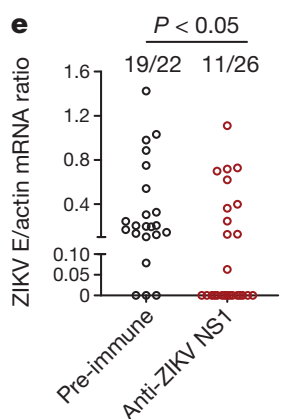

h
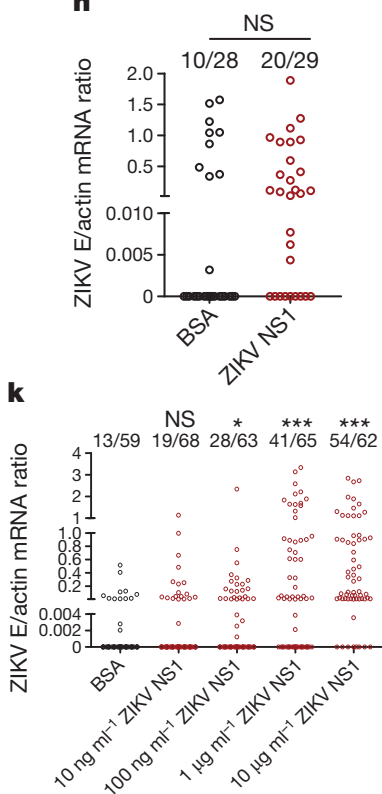

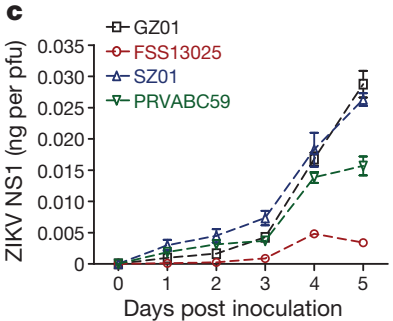

f

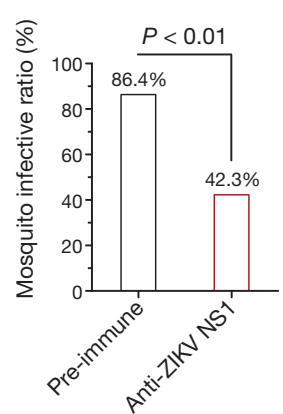

i

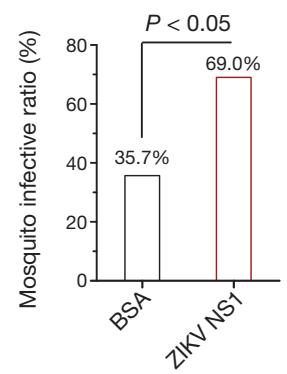

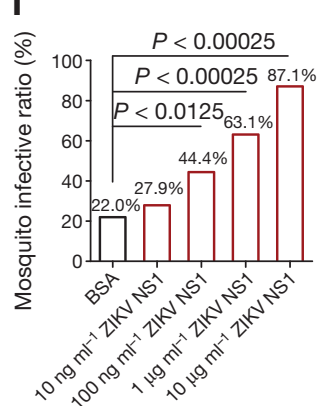

Figure 2 Differences in NS1 secretion determine ZIKV infectivity

in mosquitoes. a-c, NS1 secretability in ZIKV-infected Vero cells. Vero cells were infected with 0.01 multiplicity of infection (MOI) viruses. c, NS1 quantity normalized against average viral titre (ng per p.f.u.). The data were pooled from three independent biological replicates. d-f, Immunoblockade of NS1 in GZ01-infected supernatants reduced ZIKV acquisition. Either $10 \mu \mathrm{l}$ of mouse ZIKV NS1 antisera or $10 \mu \mathrm{l}$ pre-immune sera was mixed with supernatant from ZIKV GZ01-infected Vero cells $(500 \mu \mathrm{l})$ and fresh human blood $(500 \mu \mathrm{l})$ for in vitro membrane feeding of $A$. aegypti. The data were pooled from two independent biological replicates. g-i, Addition of NS1 to FSS13025infected supernatant increased mosquito infection. Either $10 \mu \mathrm{g}$ purified NS1 or $10 \mu \mathrm{g}$ BSA was incubated with fresh human blood $(500 \mu \mathrm{l})$ and supernatant from ZIKV FSS13025-infected Vero cells (500 $\mu \mathrm{l})$ to feed

might have evolved over time, contributing to the prevalence and spread of the virus from Asia to the Americas. To test this hypothesis, we compared the viral infectivity of two clinical ZIKV isolates of the Asian lineage, GZ01 (isolated in $2016^{13}$ ) and FSS13025 (isolated in 2010 ${ }^{14,15}$ ), in the primary urban mosquito vector $A$. aegypti. Phylogenetic analysis showed that these two strains represent the clades corresponding to the epidemic strains of ZIKV that are prevalent in the Americas (20152016) and Southeastern Asia (2007-2012) (Extended Data Fig. 1).
A. aegypti. The data were pooled from two independent biological replicates. $\mathbf{j}-\mathbf{l}$, Assessment of the threshold NS1 concentration that enhances ZIKV acquisition. Serial dilutions of purified ZIKV NS1 $(10 \mu \mathrm{l})$ were mixed with purified GZ01 ZIKV virions $(10 \mu \mathrm{l})$ and fresh human blood $(1,000 \mu \mathrm{l})$ for $A$. aegypti feeding. The data were pooled from four independent biological replicates. A final concentration of $1 \times 10^{5}$ p.f.u. $\mathrm{ml}^{-1} \mathrm{ZIKV}$ was used for mosquito oral infection $(\mathbf{d}, \mathbf{g}, \mathbf{j})$. The number at the top of each column represents infected number/total number. Each dot represents a mosquito $(\mathbf{e}, \mathbf{h}, \mathbf{k})$. Data are mean \pm s.e.m. $(\mathbf{a}-\mathbf{c}) . P$ values were determined by two-tailed Mann-Whitney test $(\mathbf{e}, \mathbf{h}, \mathbf{k})$ or two-sided Fisher's exact test $(\mathbf{f}, \mathbf{i}, \mathbf{l})$, and adjusted using Bonferroni correction to account for multiple comparisons $(\mathbf{k}, \mathbf{l})$. The $P$ value represents a comparison between BSA and other groups $(\mathbf{k}, \mathbf{l})$. $* P<0.0125, * * * P<0.00025, \mathrm{NS}$, not significant.

Thus, we exploited a 'host-mosquito' acquisition model, in which mosquitoes acquire ZIKV from a viraemic AG6 mouse ${ }^{4,16,17}$, to assess the infectivity of these ZIKV strains (Fig. 1a). Both strains exhibited similar replication kinetics in the blood of infected AG6 mice (Fig. 1b). However, the serum level of secreted NS1 was much lower in mice infected with the FSS13025 strain than the GZ01 strain (Fig. 1c). The infected mice were subjected to daily biting by the Rockefeller laboratory strain of $A$. aegypti from days 1 to 5 after mouse infection 
(Fig. 1a). Infection prevalence was substantially lower in mosquitoes that fed on FSS13025-infected mice than in those that fed on GZ01infected mice (Fig. 1d, e).

Secretion of NS1 into the serum of an infected host is a common property of many flaviviruses ${ }^{4,18-21}$. Our previous study demonstrated that circulating NS1 acquired by mosquitoes together with virions when mosquitoes feed on a viraemic mammalian host enhances viral infectivity ${ }^{4}$. NS1 antigenaemia plays a key role in maintaining the flavivirus life cycle ${ }^{4}$. We hypothesize that NS1 antigenaemia is responsible for the difference in mosquito infectivity between the GZ01 and FSS13025 strains. We therefore infected Vero cells (a monkey kidney cell line) with the American isolates GZ01 and PRVABC59 ${ }^{22}$, the SZ01 strain representing the French Polynesian clade ${ }^{23}$, and the FSS13025 strain. The three post-epidemic strains showed higher NS1 secretion than the FSS13025 strain (Fig. 2a-c), even when NS1 amount was normalized to viral titre (Fig. $2 \mathrm{c}$ ).

To determine whether NS1 secretability correlates with ZIKV infectivity in mosquitoes, we generated a mouse NS1 polyclonal antibody (Extended Data Fig. 2a). Neutralization of NS1 by antibody treatment reduced the mosquito infection prevalence of the GZ01 strain (Fig. 2d-f). The mouse NS1 antibodies did not neutralize ZIKV virions (Extended Data Fig. 3). We also tested whether the addition of NS1 protein could enhance ZIKV infectivity. A recombinant GZ01 NS1 protein was expressed and purified using a Drosophila S2 expression system (Extended Data Fig. 2b). The presence of NS1 increased the infectivity of the FSS13025 strain in mosquitoes (Fig. 2g-i). We next assessed the threshold concentration of NS1 that can effectively enhance ZIKV acquisition. Compared to bovine serum albumin (BSA), the presence of $100 \mathrm{ng} \mathrm{ml}^{-1}$ or higher concentrations of NS1 substantially increased ZIKV prevalence in A. aegypti (Fig. 2 j-1). To identify the role of animal NS1 antigenaemia in ZIKV infectivity in mosquitoes, we allowed mosquitoes to feed on viraemic AG6 mice (Extended Data Fig. 4a). The NS1 antibody treatment reduced the quantity of circulating NS1 in mouse serum (Extended Data Fig. 4b) but did not influence GZ01 ZIKV replication in AG6 mice (Extended Data Fig. 4c). The infection ratios of fed $A$. aegypti were reduced as a result of the antibodymediated neutralization of NS1 in mice (Extended Data Fig. 4d, e).

Considering the difference in secretion of NS1 between the GZ01 and FSS13025 strains (Figs 1c, 2a-c), we next attempted to identify the amino acids that are critical for NS1 secretion. We focused on the endoplasmic reticulum transmembrane region of pre-membrane (prM), the full length of envelope (E), and NS1 (prM-E-NS1) ${ }^{24}$ (Fig. 3a). There are only two sites that vary between the GZ01 and FSS13025 prM-E-NS1 proteins: residue 473 in the E protein (Val/FSS13025 to Met/GZ01) and residue 188 in the NS1 protein (Ala/FSS13025 to Val/GZ01) (Fig. 3a). We therefore individually subcloned the prM-E-NS1 regions from the GZ01 and FSS13025 strains and subsequently transfected these segments into 293T cells. Ectopic expression of the GZ01 prM-E-NS1 protein, but not the FSS13025 protein, led to abundant NS1 secretion in the supernatants of transfected cells (Fig. 3b). Mutation from valine to methionine at residue 473 of the E protein in the FSS13025 prM-E-NS1 protein did not improve NS1 secretion; however, the alanine-to-valine substitution at residue 188 of NS1 rendered NS1 secretable (Fig. 3b), demonstrating that this single mutation determines NS1 secretability. Residue 188 is located within the interface between two NS1 monomers ${ }^{25}$ (Extended Data Fig. 5a). Nonetheless, the alanine-tovaline substitution at position 188 does not influence NS1 dimerization (Extended Data Fig. 5b), suggesting that the mechanism by which the single mutation affects NS1 remains to be identified.

We next performed an alignment of the NS1 proteins from the Asian clade of ZIKV. In ZIKV isolates collected before 2012, residue 188 of the NS1 protein was alanine. However, this residue was mutated to valine in all isolates collected after 2013 (Extended Data Fig. 6), suggesting that the Asian lineage of ZIKV evolved to acquire NS1 secretability when it spread from southeastern Asia to the Southern Pacific islands around 2013. Correspondingly, Singapore reported local ZIKV transmission in
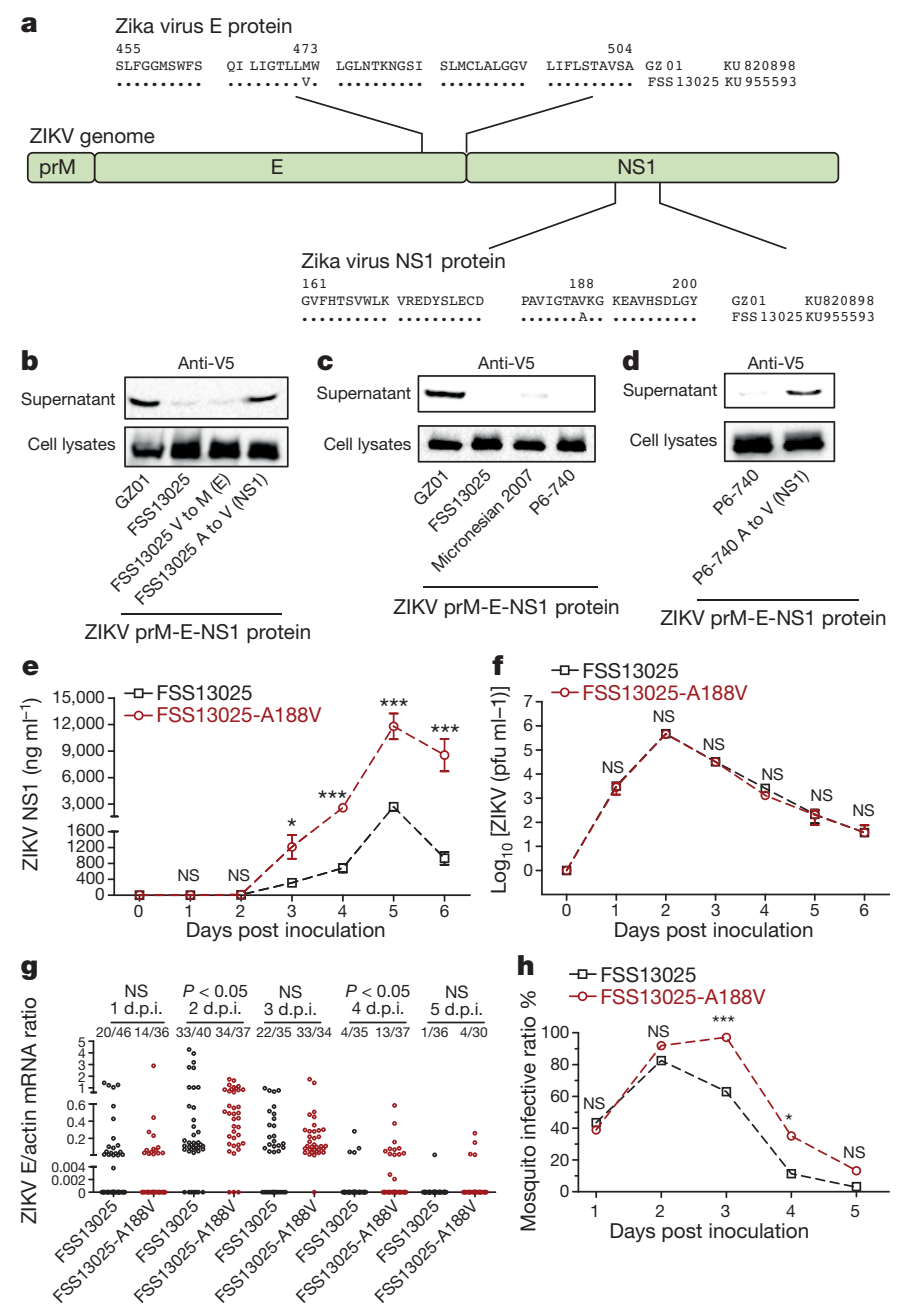

Figure 3 A single amino acid residue in NS1 determines its secretability in the Asian lineage of ZIKV. a, Comparison of the prM-E-NS1 sequences from GZ01 and FSS13025 isolates. b, Detection of NS1 secretion in ectopically expressed prM-E-NS1 proteins from the subcloned and mutated GZ01 and FSS13025 strains. c, Detection of NS1 secretion in ectopically expressed prM-E-NS1 proteins from ZIKV Asian strains. d, Mutation of alanine to valine in the NS1 protein conferred NS1 secretability on the prM-E-NS1 proteins of a Malaysia P6-740 strain. b-d, The experiments were reproduced three times with similar results. For gel source data, see Supplementary Fig. 1. e, Measurement of ZIKV NS1 antigenaemia by ELISA ( $n=10$ mice per group pooled from 5 independent biological replicates). $\mathbf{f}$, Detection of the ZIKV load in the mouse blood by plaque assay ( $n=8$ mice per group pooled from 4 independent biological replicates). $\mathbf{g}, \mathbf{h}$, Comparison of mosquito infectivity (g) and infective ratio (h) of ZIKV strains ( $n=6$ mice per group pooled from 3 independent biological replicates). The number at the top of each column represents infected number/total number. Each dot represents a mosquito (g). AG6 mice were intradermally inoculated with $1 \times 10^{4}$ p.f.u. of the FSS13025 and FSS13025-A188V strains. Data are mean \pm s.e.m. (e, f). $P$ values were determined by two-tailed Mann-Whitney test $(\mathbf{e}-\mathbf{g})$ or two-sided Fisher's exact test (h). $* P<0.05, * * * P<0.001, \mathrm{NS}$, not significant.

August 2016. The Singaporean strains stemmed from an ancestral node of the Asian lineage ${ }^{26}$. In all ZIKV isolates from Singapore, residue 188 of the NS1 protein was a valine (Extended Data Fig. 6), suggesting that the alanine-to-valine substitution may have contributed to the recent ZIKV epidemics. Therefore, we synthesized the prM-E-NS1 genes from a Malaysia P6-740 strain isolated in 1966 and a Micronesian 2007 strain and ectopically expressed these genes in 293T cells. Compared to the secretion of NS1 in the supernatants of GZ01 prM-E-NS1-transfected cells, the NS1 protein was hardly detectable in the supernatants of these 

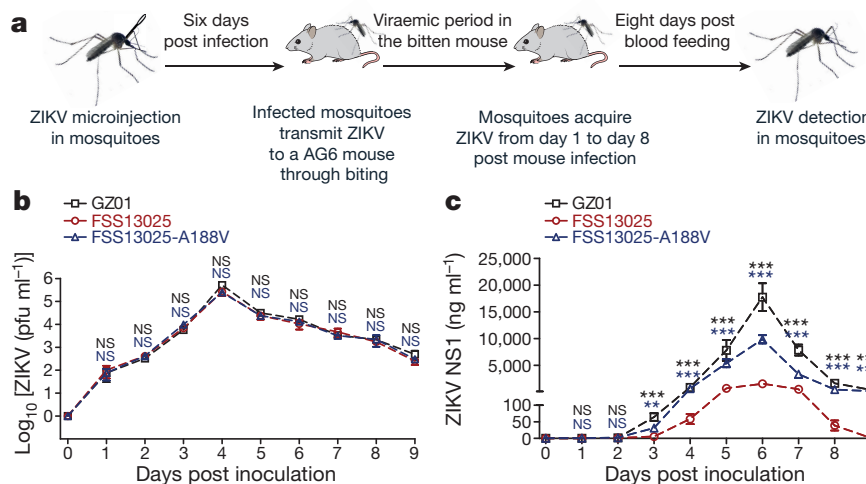
Z $\mathrm{KV}$ from day 1 to day 8 post mouse infection post mouse infection
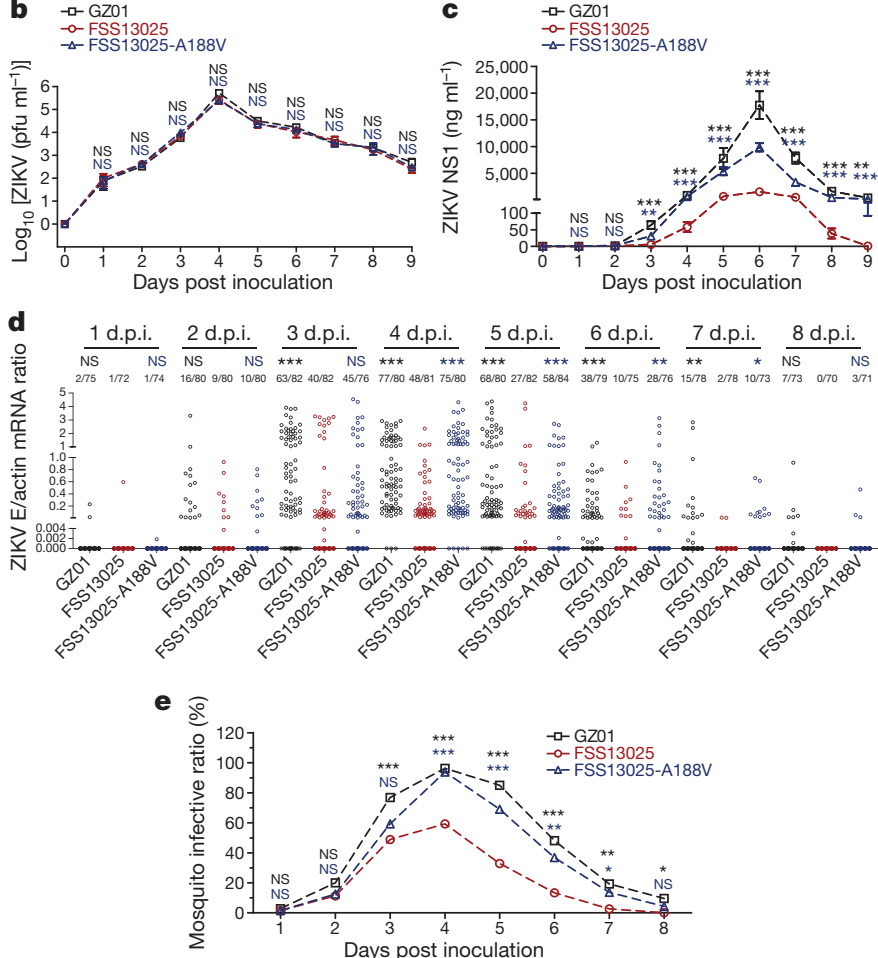

Figure 4 | A single amino acid substitution influences the prevalence of mosquito infection during the mosquito-host-mosquito life cycle. a, Schematic representation of the study design. b, Detection of ZIKV viraemia by plaque assay $(n=12$ mice per group pooled from 4 independent biological replicates). $c$, Measurements of ZIKV NS1 antigenaemia by ELISA ( $n=12$ mice per group pooled from 4 independent biological replicates). d, e, Comparison of mosquito infectivity (d) and infective ratio (e) of ZIKV strains ( $n=9$ mice per group pooled from 3 independent biological replicates). The number at the top of each column represents infected number/total number. Each dot represents a mosquito $(\mathbf{d})$. Data are mean \pm s.e.m. $(\mathbf{b}, \mathbf{c}) . P$ values were determined by two-tailed Mann-Whitney test (b-d) or two-sided Fisher's exact test (e), and adjusted using Bonferroni correction to account for multiple comparisons $(\mathbf{b}-\mathbf{e})$. The black $P$ value represents a comparison between GZ01 and FSS13025. The blue $P$ value represents a comparison between FSS13025-A188V and FSS13025. $* P<0.025$, $* * P<0.005$, $* * * P<0.0005, \mathrm{NS}$, not significant.

pre-epidemic ZIKV prM-E-NS1-expressing cells (Fig. 3c). However, the alanine-to-valine mutation in the NS1 protein of the P6-740 strain conferred NS1 secretability (Fig. 3d).

Next, we created the alanine-to-valine mutation at residue 188 of the NS1 protein in an infectious clone of the FSS13025 strain (FSS13025A $188 \mathrm{~V})^{15}$. The substitution substantially increased NS1 secretion in the supernatant of infected Vero cells (Extended Data Fig. 7a-c). The FSS13025-A188V strain showed higher ZIKV infectivity than the wild-type virus in mosquitoes (Extended Data Fig. 7d, e). In the mousemosquito acquisition model, NS1 antigenaemia was increased in the serum of FSS13025-A188V ZIKV-infected mice (Fig. 3e). Mutation of residue 188 of NS1 did not influence viral infectivity or dissemination kinetics in infected AG6 mouse blood (Fig. 3f). However, the substitution enhanced ZIKV transmission from mice to mosquitoes (Fig. 3g, h). Moreover, neutralization of NS1 with mouse ZIKV NS1 antibodies effectively reduced the prevalence of FSS13025-A188V ZIKV in mosquitoes (Extended Data Fig. 8a-d), confirming that the valine at residue
188 of NS1 has a critical role in antigenaemia, which is essential for ZIKV acquisition by mosquitoes.

We next investigated whether NS1-mediated enhancement of ZIKV infectivity influenced the prevalence of ZIKV-carrying mosquitoes using a mosquito-mouse-mosquito transmission cycle. We thoracically inoculated equal titres (5 plaque-forming units (p.f.u.)) of the GZ01, FSS13025 and FSS13025-A188V mutant strains into female $A$. aegypti mosquitoes. Six days after virus inoculation, two infected mosquitoes were allowed to feed on AG6 mice (Fig. 4a). The mosquito-bitten animals developed high-titre viraemia for all three ZIKV strains (Fig. 4b). Replication of both the GZ01 and FSS13025-A188V ZIKV strains, but not the FSS13025 strain, resulted in efficient NS1 antigenaemia (Fig. 4c). Subsequently, the infected mice were subjected to daily biting by naive $A$. aegypti from days 1 to 8 after mouse infection (Fig. 4a). Through a complete transmission cycle, the FSS13025-A188V strain showed a substantially higher prevalence of mosquito infection than did the FSS13025 strain (Fig. 4d, e), demonstrating that the valine at residue 188 is required for the generation of NS1 antigenaemia, which enhances ZIKV transmissibility from hosts to vectors.

ZIKV independently separated into the African and Asian lineages. We found that residue 188 in all ZIKV African strains was valine (Extended Data Fig. 9a). The Uganda MR766-infected Vero cells abundantly secreted NS1 (Extended Data Fig. 9b-d), implying that infection with African ZIKV might produce high NS1 antigenaemia. ZIKV has two ecologically distinct transmission cycles: a sylvatic cycle (mosquitoes to non-human primates) and a human cycle (mosquitoes to humans $)^{27}$. Knowledge of ZIKV before 2012 was almost exclusively centred on the enzootic sylvatic cycle in Africa ${ }^{27,28}$. A mosquito survey in Africa showed that ZIKV was detected in mosquitoes collected in multiple surveyed landscapes, except for indoor locations within villages ${ }^{28}$. Therefore, we hypothesize that the presence of a valine at residue 188 of NS1 might bestow on the African lineage of ZIKV a highly efficient enzootic or epizootic transmission cycle ${ }^{27,28}$. Other unknown factor(s) might prevent African ZIKV from efficiently establishing a human infection cycle. However, the Asian strains may have evolved to target humans with high infectivity; indeed, one such strain caused a medium-scale epidemic in Micronesia in 2007 ${ }^{1,9}$. The alanine-to-valine substitution at position 188 further improved ZIKV transmission efficiency from humans to mosquitoes and thus increased its prevalence in mosquitoes. Our data offer a potential explanation for the recent re-emergence of ZIKV, and suggest that co-evolution of arboviruses with their hosts or vectors contributes to their long-term existence and occasional widespread re-emergence.

Online Content Methods, along with any additional Extended Data display items and Source Data, are available in the online version of the paper; references unique to these sections appear only in the online paper.

Received 30 July 2016; accepted 10 April 2017. Published online 17 May 2017.

1. Musso, D. \& Gubler, D. J. Zika virus. Clin. Microbiol. Rev. 29, 487-524 (2016).

2. Musso, D., Nilles, E. J. \& Cao-Lormeau, V. M. Rapid spread of emerging Zika virus in the Pacific area. Clin. Microbiol. Infect. 20, 0595-0596 (2014).

3. Enfissi, A., Codrington, J., Roosblad, J., Kazanii, M. \& Rousset, D. Zika virus genome from the Americas. Lancet 387, 227-228 (2016).

4. Liu, J. et al. Flavivirus NS1 protein in infected host sera enhances viral acquisition by mosquitoes. Nat. Microbiol. 1, 16087 (2016).

5. Marchette, N. J., Garcia, R. \& Rudnick, A. Isolation of Zika virus from Aedes aegypti mosquitoes in Malaysia. Am. J. Trop. Med. Hyg. 18, 411-415 (1969).

6. Oehler, E. et al. Zika virus infection complicated by Guillain-Barre syndromecase report, French Polynesia, December 2013. Euro Surveill. 19, 4-6 (2014).

7. Cordeiro, M. T., Pena, L. J., Brito, C. A., Gil, L. H. \& Marques, E. T. Positive IgM for Zika virus in the cerebrospinal fluid of 30 neonates with microcephaly in Brazil. Lancet 387, 1811-1812 (2016).

8. Faye, O. et al. Molecular evolution of Zika virus during its emergence in the 20th century. PLoS Negl. Trop. Dis. 8, e2636 (2014).

9. Duffy, M. R. et al. Zika virus outbreak on Yap Island, Federated States of Micronesia. N. Engl. J. Med. 360, 2536-2543 (2009)

10. Cao-Lormeau, V. M. et al. Zika virus, French Polynesia, South Pacific, 2013. Emerg. Infect. Dis. 20, 1085-1086 (2014). 
11. Brault, A. C. et al. Venezuelan equine encephalitis emergence: enhanced vector infection from a single amino acid substitution in the envelope glycoprotein. Proc. Natl Acad. Sci. USA 101, 11344-11349 (2004).

12. Tsetsarkin, K. A., Vanlandingham, D. L., McGee, C. E. \& Higgs, S. A single mutation in chikungunya virus affects vector specificity and epidemic potential. PLoS Pathog. 3, e201 (2007).

13. Zhang, F. C., Li, X. F., Deng, Y. Q., Tong, Y. G. \& Qin, C. F. Excretion of infectious Zika virus in urine. Lancet Infect. Dis. 16, 641-642 (2016).

14. Heang, V. et al. Zika virus infection, Cambodia, 2010. Emerg. Infect. Dis. 18, 349-351 (2012).

15. Shan, C. et al. An infectious cDNA clone of Zika virus to study viral virulence, mosquito transmission, and antiviral inhibitors. Cell Host Microbe 19, 891-900 (2016).

16. Cheng, G., Liu, Y., Wang, P. \& Xiao, X. Mosquito defense strategies against viral infection. Trends Parasitol. 32, 177-186 (2016).

17. Lazear, H. M. et al. A mouse model of Zika virus pathogenesis. Cell Host Microbe 19, 720-730 (2016)

18. Muller, D. A. \& Young, P. R. The flavivirus NS1 protein: molecular and structural biology, immunology, role in pathogenesis and application as a diagnostic biomarker. Antiviral Res. 98, 192-208 (2013).

19. Young, P. R., Hilditch, P. A., Bletchly, C. \& Halloran, W. An antigen capture enzyme-linked immunosorbent assay reveals high levels of the dengue virus protein NS1 in the sera of infected patients. J. Clin. Microbiol. 38, 1053-1057 (2000).

20. Macdonald, J. et al. NS1 protein secretion during the acute phase of West Nile virus infection. J. Virol. 79, 13924-13933 (2005).

21. Takamatsu, Y. et al. NS1' protein expression facilitates production of Japanese encephalitis virus in avian cells and embryonated chicken eggs. J. Gen. Virol. 95, 373-383 (2014).

22. Lanciotti, R. S., Lambert, A. J., Holodniy, M., Saavedra, S. \& Signor, L. del. C. Phylogeny of Zika virus in Western Hemisphere. 2015. Emerg. Infect. Dis. 22, 933-935 (2016).

23. Deng, Y. Q. et al. Isolation, identification and genomic characterization of the Asian lineage Zika virus imported to China. Sci. China Life Sci. 59, 428-430 (2016).

24. Zhang, Y. et al. Structures of immature flavivirus particles. EMBO J. 22, 2604-2613 (2003).

25. Song, H., Qi, J., Haywood, J., Shi, Y. \& Gao, G. F. Zika virus NS1 structure reveals diversity of electrostatic surfaces among flaviviruses. Nat. Struct. Mol. Biol. 23, 456-458 (2016).

26. Maurer-Stroh, S. et al. South-east Asian Zika virus strain linked to cluster of cases in Singapore, August 2016. Euro Surveill. 21, 2 (2016).
27. Buechler, C. R. et al. Seroprevalence of Zika virus in wild African green monkeys and baboons. mSphere 2, e00392-16 (2017).

28. Diallo, D. et al. Zika virus emergence in mosquitoes in southeastern Senegal, 2011. PLoS One 9, e109442 (2014).

Supplementary Information is available in the online version of the paper.

Acknowledgements This work was funded by grants from the National Key Research and Development Plan of China (2016YFD0500400, 2016ZX10004001-008, 2017ZX09101-005, 2016YFC1201000, 2016YFD0500300), the National Natural Science Foundation of China $(81422028,81571975,81522025,31300600)$, the National Key Basic Research Program of China (2013CB911500, SZSTI-JCYJ2016033111585 3521), the National Institutes of Health of the United States (R01AI087856, Al103807), and Shenzhen San-Ming Project for prevention and research on vector-borne diseases. G.C. is a Newton Advanced Fellow awarded by the Academy of Medical Sciences and the Newton Fund. G.C. is also a Janssen Investigator at Tsinghua University. P.-Y.S. was supported by a University of Texas Medical Branch (UTMB) startup award and a University of Texas STARs Award. We thank the core facilities of the Center for Life Sciences and Center of Biomedical Analysis (Tsinghua University) for technical assistance.

Author Contributions G.C. and P.-Y.S. designed the experiments and wrote the manuscript; Y.L., J.L., S.D. and C.S. performed the majority of the experiments and analysed the data; K.N. and R.Z. assisted in RNA isolation and qPCR detection; C.S., X.-F.L., C.-F.O. and P.-Y.S. provided the ZIKV strains; X.-F.L., R.Z., T.W., C.-F.Q. and P.W. contributed experimental suggestions and strengthened the writing of the manuscript. All authors reviewed, critiqued and provided comments on the text.

Author Information Reprints and permissions information is available at www.nature.com/reprints. The authors declare no competing financial interests. Readers are welcome to comment on the online version of the paper. Publisher's note: Springer Nature remains neutral with regard to jurisdictional claims in published maps and institutional affiliations. Correspondence and requests for materials should be addressed to G.C. (gongcheng@mail.tsinghua. edu.cn) and P.-Y.S. (peshi@utmb.edu).

Reviewer Information Nature thanks S. Higgs, W. Reisen and the other anonymous reviewer(s) for their contribution to the peer review of this work. 


\section{METHODS}

Ethics statement. Human blood for mosquito feeding was taken from healthy donors who provided written informed consent. The collection of human blood samples was approved by the local ethics committee at Tsinghua University.

Mice, mosquitoes, cells and viruses. AG6 mice were donated by the Institute Pasteur of Shanghai, Chinese Academy of Sciences. The mice were bred and maintained under a specific pathogen-free animal facility at Tsinghua University. Groups of age- and sex-matched AG6 mice, 6-8 weeks of age, were used for the animal studies ${ }^{4}$. All experiments were approved by and performed under the guidelines of the Experimental Animal Welfare and Ethics Committee of Tsinghua University. A. aegypti (Rockefeller strain) were reared in a low-temperature, illuminated incubator (Model 818, Thermo Electron Corporation) at $28^{\circ} \mathrm{C}$ and $80 \%$ humidity according to standard rearing procedures ${ }^{29}$. The ZIKV GZ01 (KU820898), PRVABC59 (KU501215), SZ01 (KU866423), MR766 (NC_012532) and FSS13025 (KU955593) ${ }^{15}$ strains were grown in Vero cells for blood meals ${ }^{4}$. The Drosophila melanogaster S2 cell line was cultured in Schneider's medium with 10\% heat-inactivated fetal bovine serum and 1\% antibiotic-antimycotic (15240-062, Invitrogen). The Vero cells and 293T cells were maintained in Dulbecco's modified Eagle's medium (DMEM, Gibco) supplemented with $10 \%$ heat-inactivated fetal bovine serum (16000-044, Gibco). For ZIKV infection, the Vero cells were maintained in VP-SFM medium (11681-020, Gibco). The Vero and 293T cell lines were purchased from the ATCC (CCL81, CRL-3216).The Drosophila S2 cell line was sourced from a Drosophila expression system of Invitrogen (R690-07). None of these cell lines were found in the database of commonly misidentified cell lines maintained by ICLAC and NCBI Biosample. All these cell lines were authenticated by ATCC or Invitrogen and do not have mycoplasma contamination. ZIKV was titred by a plaque formation (p.f.u.) assay ${ }^{29}$.

Antibodies. The ZIKV NS1 genes were amplified from the cDNA of infected cells and cloned into a pET-28a (+) expression vector (69864, Millipore). The cloning primers are presented in Extended Data Table 1. Recombinant NS1 proteins were expressed in the Escherichia coli BL21 DE3 strain in an insoluble form in inclusion bodies. The proteins were then solubilized by $8 \mathrm{M}$ urea and purified with a TALON Purification Kit (635515, Clontech). Mouse antisera were produced via inoculation with recombinant ZIKV NS1 with three boosts. Polyclonal antibodies were purified from the immunized antisera using protein $\mathrm{A} / \mathrm{G}$ agarose (20423, Thermo). The anti-V5-HRP antibody for tag detection was purchased from Invitrogen (R96125, Thermo)

Expression of prM-E-NS1 protein in human cells. The $p r M-E-N S 1$ regions (base pairs 820-3,438 in GZ01 KU820898; 927-3,545 in FSS13025 KU955593) were amplified from cDNA of infected cells. The prM-E-NS1 genes from the P6-740 strain (base pairs 820-3,438, HQ234499) and the Micronesian 2007 strain (base pairs 820-3,438, EU545988) were commercially synthesized (Genescript). All the genes were subcloned into a pcDNA3.1/V5-His/TOPO DNA vector (K4800-01, Invitrogen), and subsequently transfected using X-treme Gene HP DNA transfection reagent (06366236001, Roche) for ectopic expression in 293T cells. The amount of NS1 in the supernatants of the 293T cells was measured $48 \mathrm{~h}$ after transfection. The cloning primers are shown in Extended Data Table 1.

ZIKV NS1 protein generation in a Drosophila expression system. The ZIKV NS1 gene was cloned into a pMT/BiP/V5-His A vector (V4130-20, Invitrogen) for expression in Drosophila S2 cells. The cloning primers are shown in Extended Data Table 1. The procedure for ZIKV NS1 expression and purification in a Drosophila expression system has been described previously ${ }^{4,29}$.

ZIKV NS1 detection by ELISA. Secretion of ZIKV NS1 into Vero cell supernatant and mouse serum was measured using a ZIKV NS1 Antigen ELISA Kit (ZIKVNS1-EK, BioFront MonoTrace). The experiment was performed according to the kit's manual. Samples were diluted to the range of quantification of the kit using the sample dilution buffer provided in the kit. Standard materials for the generation of a standard curve were also provided in the kit. The measured amount of ZIKV NS1 matched the linearity in the assay. Optical density (OD) was measured at $450 \mathrm{~nm}$ with an ELISA reader (Varioskan Flash Multimode Reader, Thermo Scientific). The detection limit of NS1 protein by ELISA is $0.1 \mathrm{ng} \mathrm{ml}^{-1}$.

Thoracic inoculation of ZIKV in mosquitoes. Detailed procedures for microinjection in mosquitoes have been described previously ${ }^{4,29}$. In brief, female A. aegypti mosquitoes were anaesthetized on a cold tray, and subsequently a certain titre (5 p.f.u.) of ZIKV was microinjected into the mosquito thoraxes. The infected mosquitoes were used for ZIKV transmission to AG6 mice by a blood meal.

Membrane blood feeding. Fresh human blood was placed in heparin-coated tubes (367884, BD Vacutainer) and centrifuged at $1,000 \mathrm{~g}$ and $4^{\circ} \mathrm{C}$ for $10 \mathrm{~min}$ to separate the plasma from the blood cells. The plasma was collected and heat-inactivated at $55^{\circ} \mathrm{C}$ for $60 \mathrm{~min}$. The separated blood cells were washed three times with PBS to remove the anticoagulant. The cells were then resuspended in the heat-inactivated plasma. The donor sera were negative for the E and NS1 proteins of JEV, DENV and ZIKV. Either purified proteins or antibodies were mixed with the ZIKV-infected supernatant and human blood for mosquito oral feeding via a Hemotek system (6W1, Hemotek Limited). Engorged female mosquitoes were transferred into new containers and maintained under standard conditions for an additional 8 days. The mosquitoes were subsequently killed for further analysis.

Mosquito feeding on infected mice. Eight to ten female A. aegypti mosquitoes were separated into a netting-covered container for blood feeding. The mosquitoes were starved for $24 \mathrm{~h}$ before engorgement. ZIKV-infected AG6 mice were anaesthetized and placed on top of the container. The mosquitoes were allowed to feed on the mice for $30 \mathrm{~min}$ in the dark. After being anaesthetized using ice, the engorged mosquitoes were transferred to new containers and maintained under standard conditions for an additional 8 days. The mosquitoes were subsequently killed for further analysis.

Purification of infectious ZIKV virions. The purification of flaviviral infectious virions has been described previously ${ }^{4}$. In brief, supernatant from GZ01-infected Vero cells was collected 5 days after infection. Cell fragments were removed by centrifugation at $25,000 \mathrm{~g}$ and $4^{\circ} \mathrm{C}$ for $20 \mathrm{~min}$. Subsequently, the supernatant was carefully transferred into a clean centrifuge tube and centrifuged at $25,000 \mathrm{~g}$ and $4^{\circ} \mathrm{C}$ for an additional $6 \mathrm{~h}$ to pellet the virions. The precipitated virions were washed twice and then solubilized in VP-SFM medium (11681-020, Gibco). Insoluble material was removed by an extra centrifugation step at $12,000 \mathrm{~g}$ and $4^{\circ} \mathrm{C}$ for $2 \mathrm{~min}$. The virions in the VP-SFM medium were aliquoted and stored at $-80^{\circ} \mathrm{C}$.

Viral genome quantification by TaqMan qPCR. Total RNA was isolated from homogenized mosquitoes using an RNeasy Mini Kit (74106, Qiagen) and reverse-transcribed into cDNA using an iScript cDNA synthesis kit (170-8890, Bio-Rad). Viral genomes were quantified via TaqMan qPCR amplification of ZIKV genes, normalized against $A$. aegypti actin (AAEL011197). The detection limit of ZIKV E/Actin mRNA ratio is 0.001 . The primers and probes used for this analysis are shown in Extended Data Table 1.

Determination of the virus titre in infected mice by plaque assay. Blood samples were collected from the tail veins of infected mice in $0.4 \%$ sodium citrate and were centrifuged for $5 \mathrm{~min}$ at $6,000 \mathrm{~g}$ and $4{ }^{\circ} \mathrm{C}$ to isolate plasma. The presence of infectious viral particles in the plasma was determined using a plaque assay ${ }^{4}$ The detection limit in the plaque assay is 10 p.f.u. $\mathrm{ml}^{-1}$ in cell supernatant, and 100 p.f.u. $\mathrm{ml}^{-1}$ in serum.

RNA transcription, transfection and virus recovery. The pFLZIKV-FSS13025 and pFLZIKV-FSS13025-A188V ${ }^{15}$ plasmids were amplified in E. coli Top10 (C404010, Invitrogen) and purified using MaxiPrep PLUS (12965, Qiagen). For in vitro transcription, $10 \mu \mathrm{g}$ plasmid was linearized with the restriction enzyme ClaI. A mMESSAGE mMACHINE T7 kit (AM1344, Ambion) was used for in vitro transcription of RNA in a $20 \mu \mathrm{l}$ reaction with an additional $1 \mu \mathrm{l}$ of $30 \mathrm{mM}$ GTP solution. The RNA was precipitated with lithium chloride and quantified by spectrophotometry. Subsequently, the RNA was transfected into Vero cells seeded in a T175 flask with DMEM medium and incubated at $37^{\circ} \mathrm{C}$ and $5 \% \mathrm{CO}_{2}$ using Lipofectamine 3000 reagent (L3000015, Invitrogen). Recombinant viruses in cell culture media were harvested 4 days after transfection, stored in aliquots at $-80^{\circ} \mathrm{C}$, and subjected to further investigation.

Statistics. Animals were randomly allocated into different groups. Mosquitoes that died before measurement were excluded from analysis. The investigators were not blinded to allocation during the experiments or to the outcome assessment. No statistical methods were used to predetermine sample size. Descriptive statistics have been provided in the figure legends. Kruskal-Wallis analysis of variance was conducted to detect any significant variation among replicates. If no significant variation was detected, the results were pooled for further comparison. Given the nature of the experiments and the types of samples, differences between continuous variables were assessed with a non-parametric Mann-Whitney test. Differences in mosquito infective rates were analysed using Fisher's exact test. $P$ values were adjusted using the Bonferroni correction to account for multiple comparisons. All analyses were performed using GraphPad Prism statistical software.

Data availability. Source Data for the main and Extended Data Figures are provided in the online version of this paper. All other data are available from the corresponding authors upon reasonable request.

29. Liu, Y. et al. Transmission-blocking antibodies against mosquito C-type lectins for dengue prevention. PLoS Pathog. 10, e1003931 (2014).

30. Brown, W. C. et al. Extended surface for membrane association in Zika virus NS1 structure. Nat. Struct. Mol. Biol. 23, 865-867 (2016). 


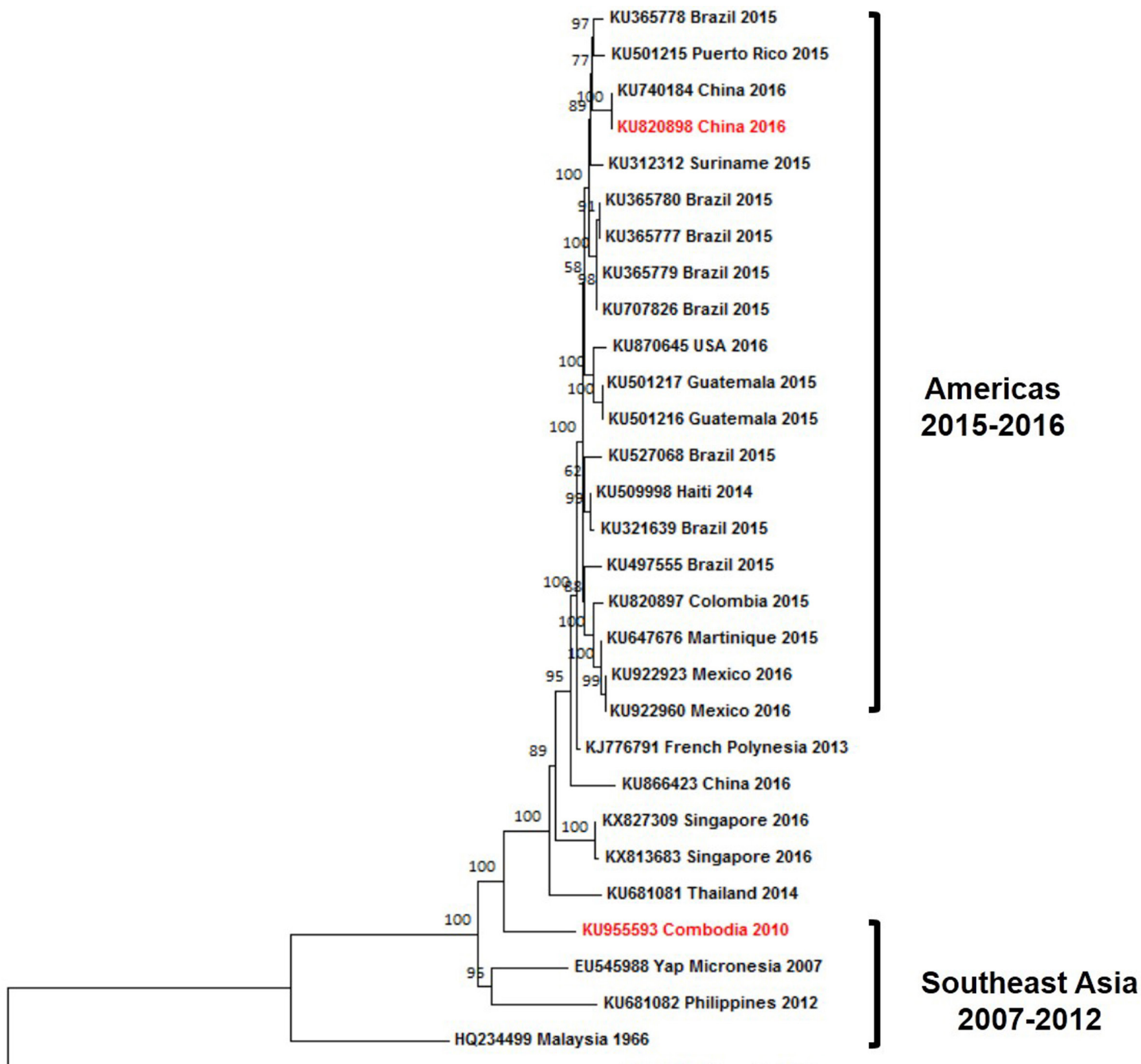

NC 012532 Uganda 1947

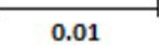

Extended Data Figure 1 | Phylogenetic analysis of ZIKV isolates in the Asian lineage. The tree was constructed using the neighbour-joining method based on the alignment of ZIKV sequences in the Asian lineage. The bootstrap values of 2,000 replicates are indicated on the branch nodes. 
a

\section{Anti-ZIKV NS1 Pre-immune}
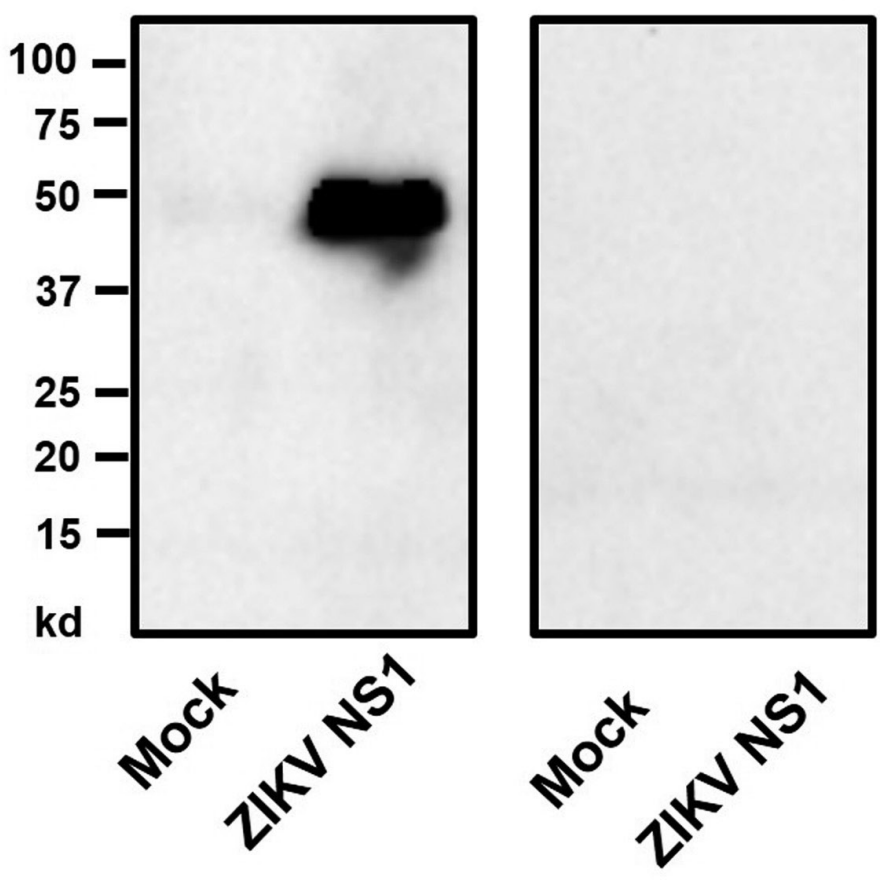

b

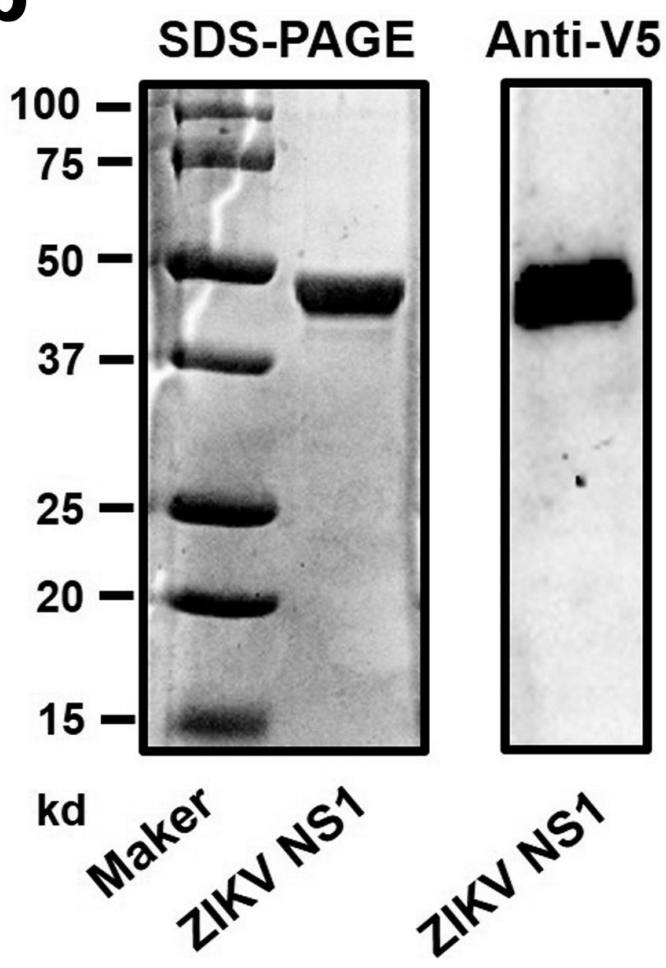

Extended Data Figure 2 | Generation of mouse anti-ZIKV NS1 polyclonal antibody and purification of the GZ01 ZIKV NS1 protein from Drosophila S2 cells. a, Generation of mouse anti-ZIKV NS1 polyclonal antibody by immunization with purified GZ01 ZIKV NS1 recombinant protein expressed in E. coli. The antibody was validated by probing an S2-expressed ZIKV NS1 recombinant protein. The same samples probed by mouse pre-immune antibody served as a negative control. b, Purification of the GZ01 ZIKV NS1 protein from Drosophila S2 cells. Full-length NS1 was cloned into the pMT/BiP/V5-His A expression vector. Recombinant ZIKV NS1 protein was expressed in Drosophila S2 cells and purified using a cobalt-His column (left). Protein quality was evaluated by western blotting with an anti-V5 antibody (right). a, b, The experiments were reproduced three times with similar results. For gel source data, see Supplementary Fig. 1. 


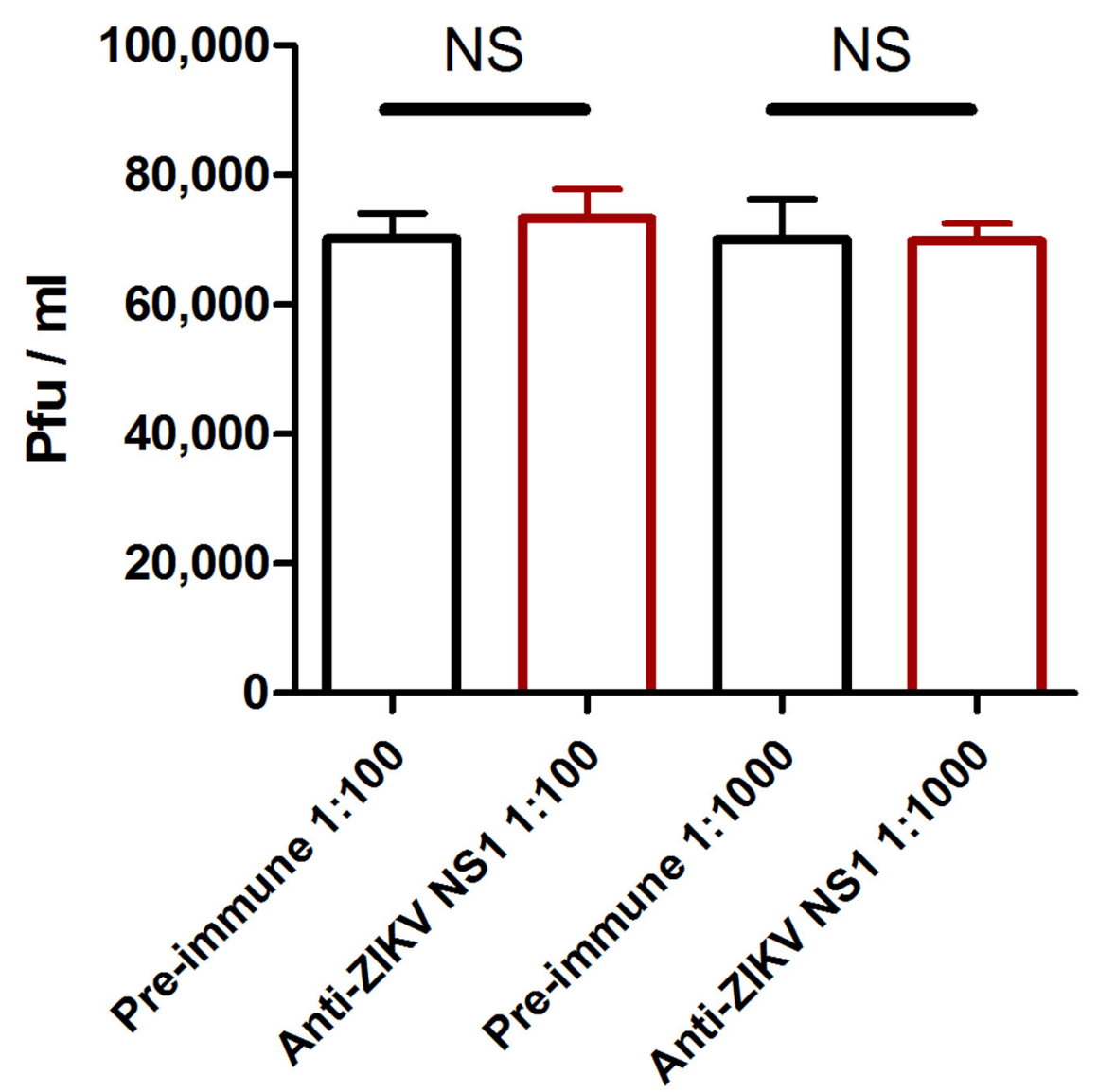

Extended Data Figure 3 | Anti-ZIKV NS1 antibodies did not neutralize ZIKV virions. We premixed two dilutions of mouse ZIKV NS1 polyclonal antisera (1:100 and 1:1,000) with purified ZIKV GZ01 infectious virions. The same amount of pre-immune serum served as a negative control. After a 30-min incubation, the infectivity of the antibody-virion mixture

was determined by plaque assay on Vero cells. The values in the graph represent the mean \pm s.e.m. $P$ values were determined by two-tailed Mann-Whitney test. The data were combined from three biological repeats. NS, not significant. 
a

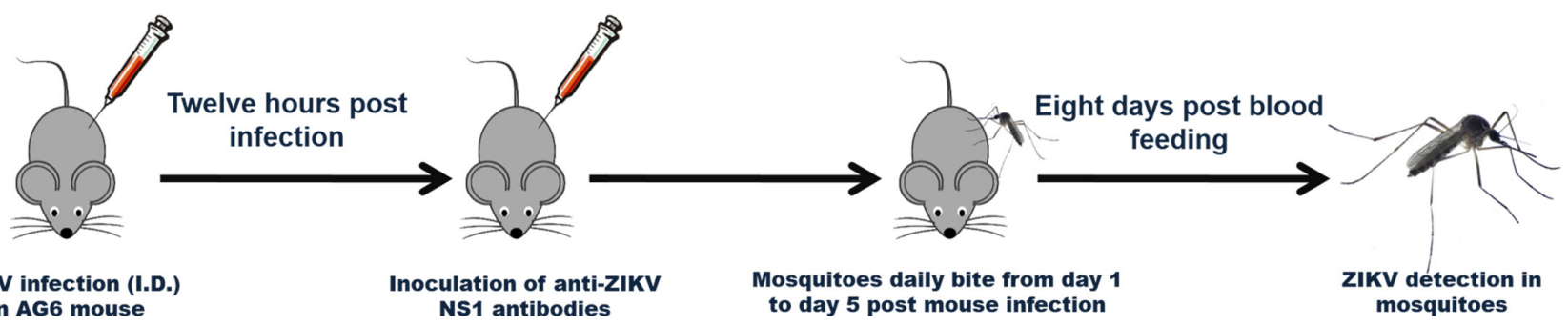

ZIKV infection (I.D.)

to day 5 post mouse infection

b

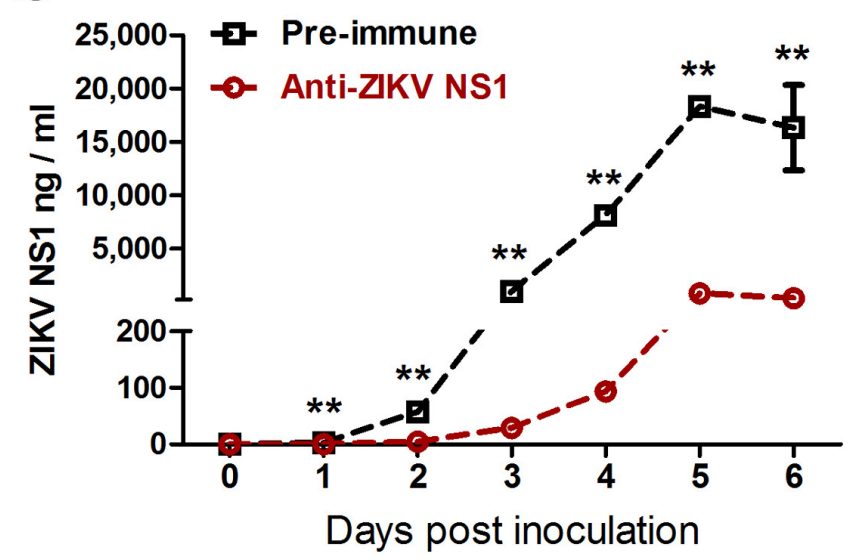

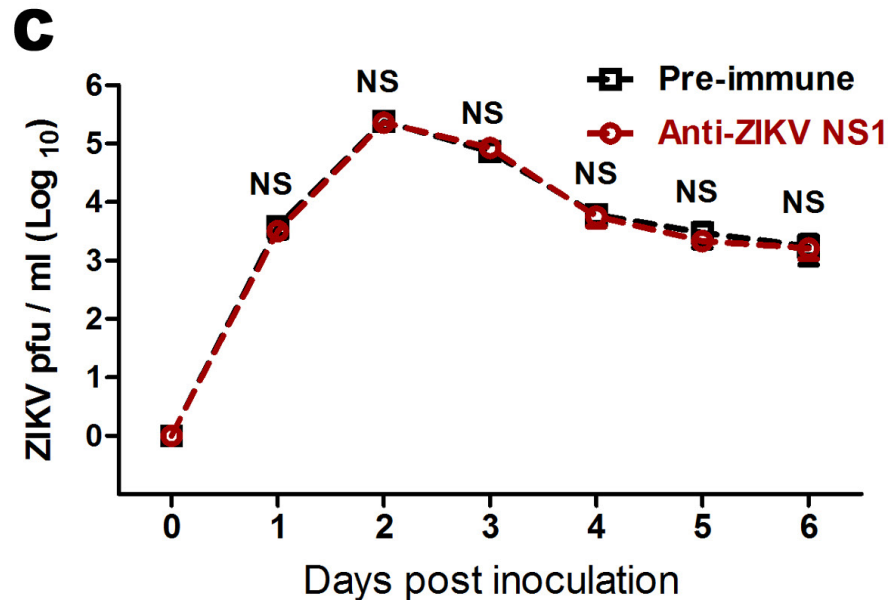

e

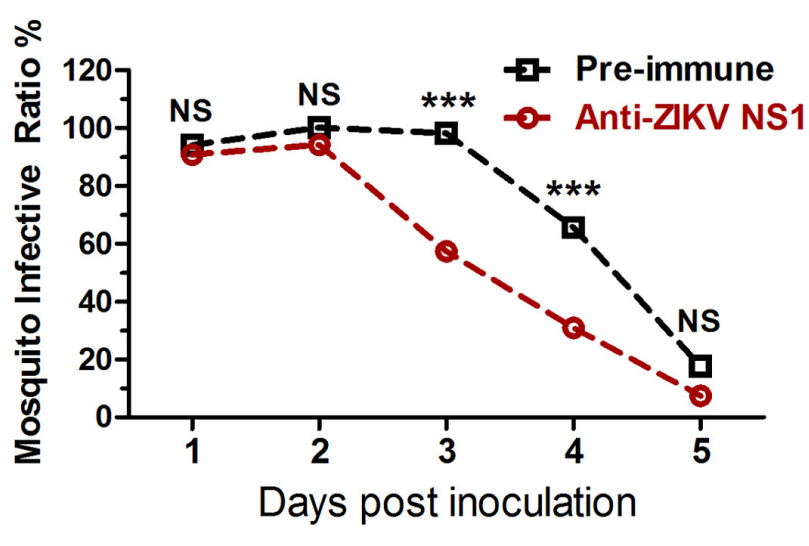

Extended Data Figure 4 | Passive transfer of ZIKV NS1 antibodies into infected AG6 mice prevented ZIKV acquisition by $A$. aegypti. a, Schematic representation of the study design. AG6 mice were intradermally infected with $1 \times 10^{4}$ p.f.u. of the ZIKV GZ01 strain. Subsequently, $100 \mu \mathrm{l}$ of a mouse ZIKV NS1 antibody was intraperitoneally inoculated into the mice $12 \mathrm{~h}$ after infection. The same amount of preimmune serum was inoculated as a mock control. After $12 \mathrm{~h}$ of antibody dissemination, the infected mice were subjected to daily biting by female A. aegypti from days 1 to 5 after mouse infection. The mouse-blood-fed mosquitoes were reared for an additional 8 days for ZIKV detection. b, ZIKV NS1 measurement by ELISA ( $n=6$ mice per group pooled from 3 independent biological replicates). Mouse serum was collected to quantify ZIKV NS1 protein from days 0 to 6 after mouse infection. c, Detection of the ZIKV load in the blood of the infected mice ( $n=8$ mice per group pooled from 4 independent biological replicates). The presence of infectious ZIKV particles in blood plasma was determined by plaque assay from days 0 to 6 after mouse infection. $\mathbf{d}, \mathbf{e}$, Immunoblockade of ZIKV NS1 in infected AG6 mice reduced the infection of fed A. aegypti ( $n=6$ mice per group pooled from 3 independent biological replicates). The number of infected mosquitoes relative to the total number of mosquitoes is shown at the top of each column. Each dot represents a mosquito (d). The data are represented as the percentage of mosquito infection (e). Data are mean \pm s.e.m. (b, c). $P$ values were determined by two-tailed Mann-Whitney test (b-d) or two-sided Fisher's exact test (e). $* * P<0.01, * * * P<0.001, \mathrm{NS}$, not significant. 


\section{RESEARCH LETTER}

a
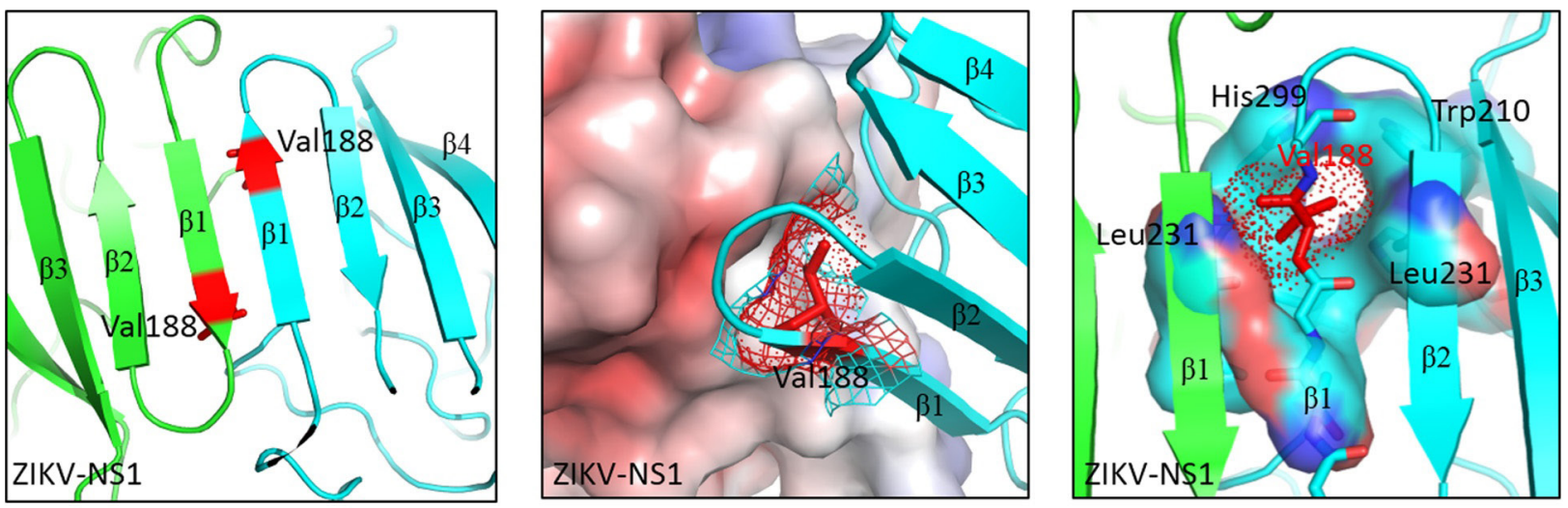

b

\section{Anti-V5}

\section{Cell lysates Dimer}

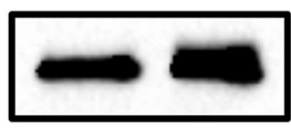

\section{Cell lysates Monomer}
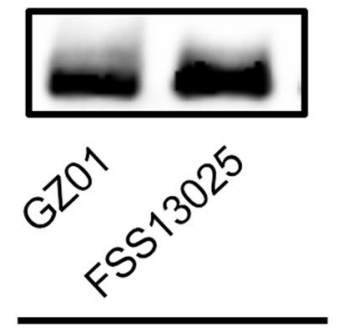

\section{ZIKV prM-E-NS1 protein}

Extended Data Figure 5 | Modelling the location of residue 188 of ZIKV NS1 and assessing NS1 dimer formation in cell lysates. a, Modelling the location of residue 188 of ZIKV NS1. Modelling was based on the ZIKV NS1 structure (PDB: $5 \mathrm{~K} 6 \mathrm{~K})^{30}$. Residue 188 is located within the interface of a NS1 dimer (contoured red), which is formed by hydrophobic interactions. Residue 188 is deep within the hydrophobic pocket formed by Trp210, Leu23, His299 and Leu231. b. Assessing NS1 dimer formation

in the lysates of cells transfected with GZ01 or FSS13025 prM-E-NS1 recombinant plasmids. The cell lysates, with or without heating treatment, were used for assessing NS1 monomers and dimers, respectively, by western blotting with an anti-V5 antibody. The experiment was repeated three times with similar results. For gel source data, see Supplementary Fig. 1. 


\section{The ZIKV Asian Lineage}

171

188

VREDYSLECD PAVIGTAVKG KEAVHSDLGY WIESEKNDTW RLKRAHLIEM KU922960_Mexico_2016

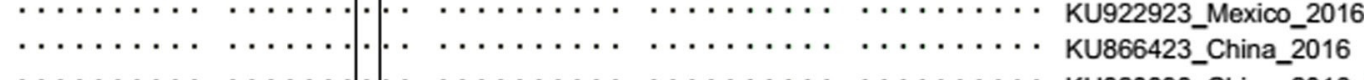

$\ldots \ldots \ldots \ldots \ldots \ldots \ldots \ldots \ldots \ldots$ KU820898_China_2016

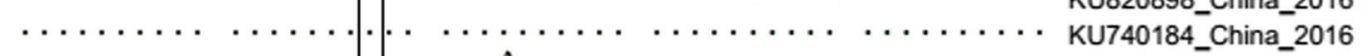

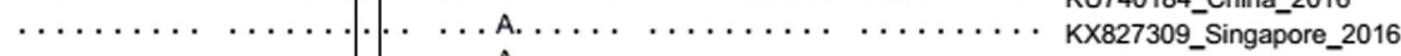

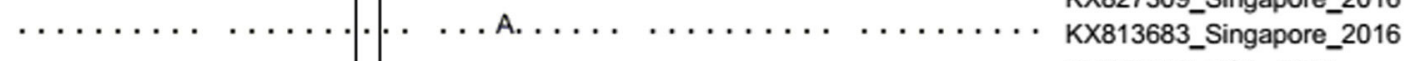

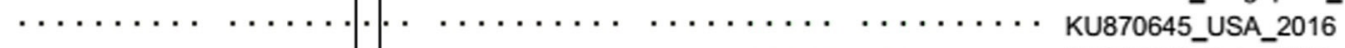

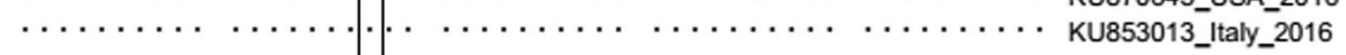

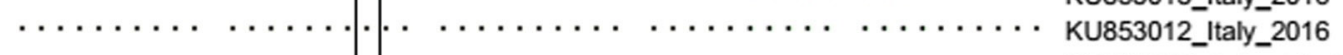

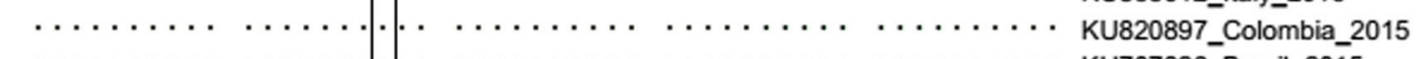

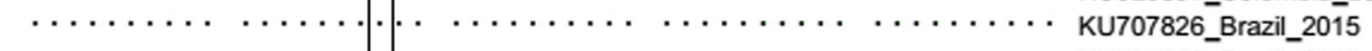

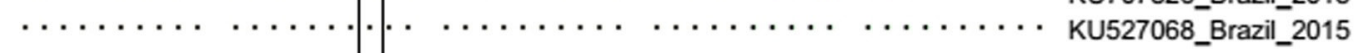

$\ldots \ldots \ldots \ldots \ldots \ldots \ldots \ldots \ldots$ KU497555_Brazil_2015

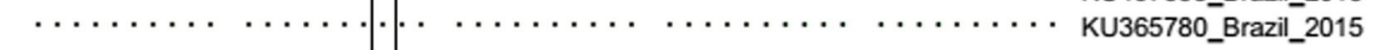

$\ldots \ldots \ldots \ldots \ldots \ldots \ldots \ldots \ldots \ldots \ldots \ldots$ KU36579_Brazil_2015

$\ldots \ldots \ldots \ldots \ldots \ldots \ldots \ldots \ldots \ldots$ KU365778_Brazil_2015

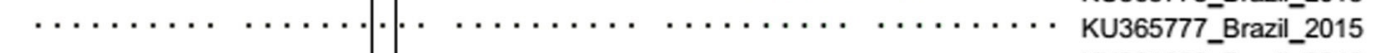

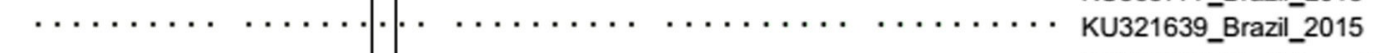

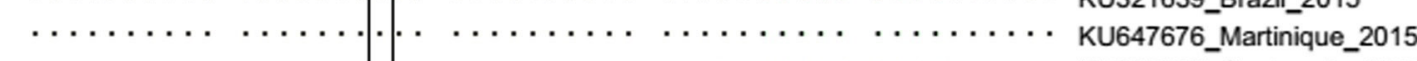

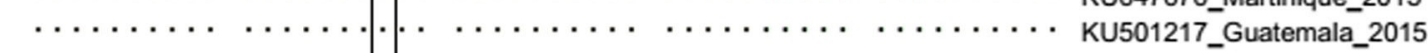

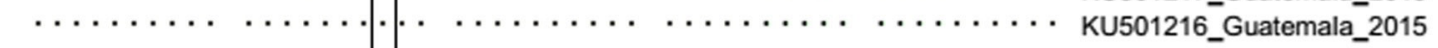

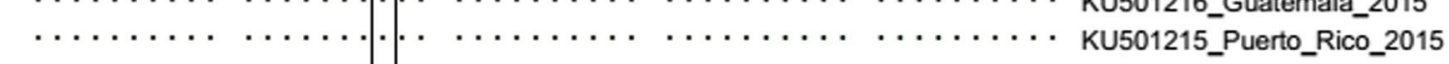

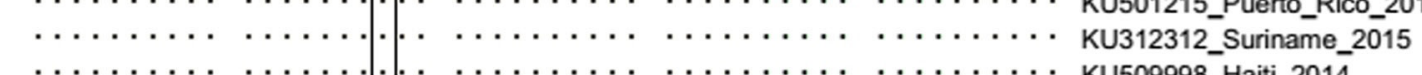

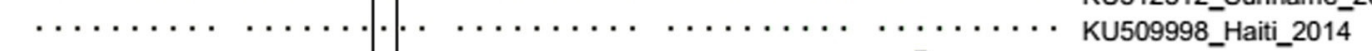

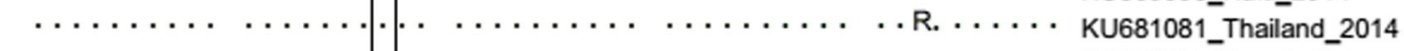

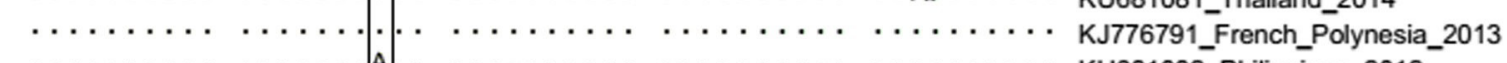

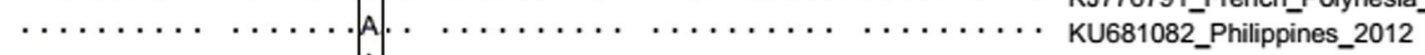

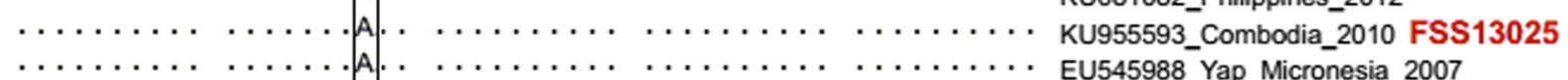

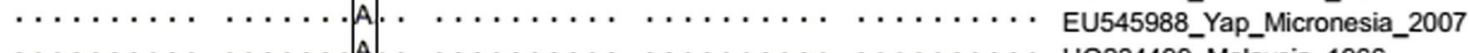

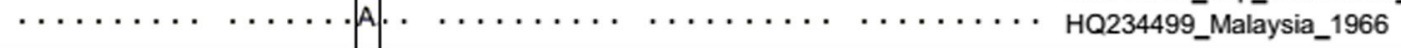

Extended Data Figure 6 | Sequence alignment of NS1 sequences in ZIKV isolates of the Asian lineage. 
a

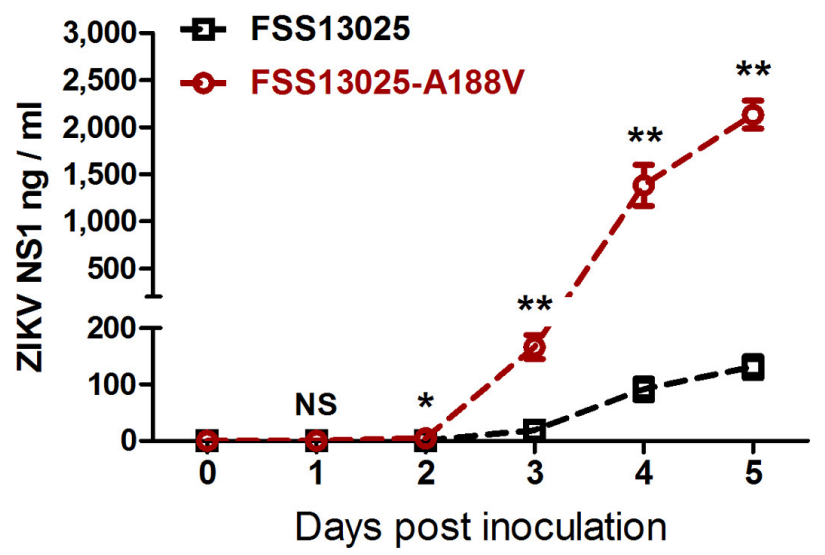

C

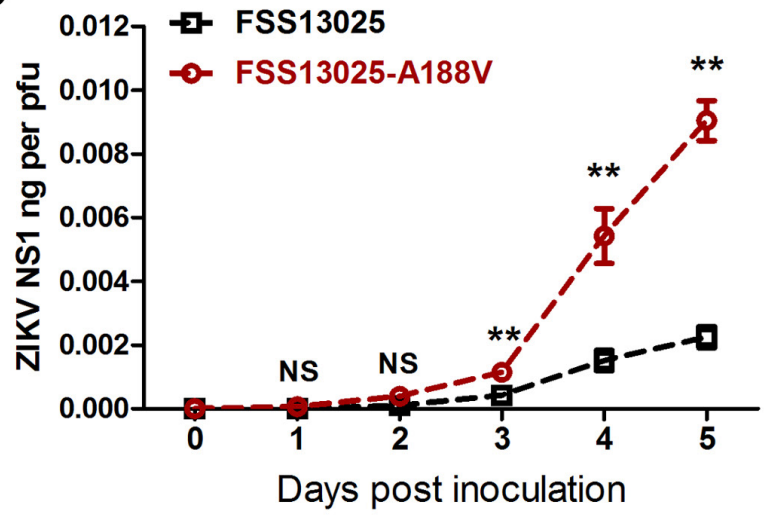

Extended Data Figure $7 \mid$ A single amino acid substitution enhanced NS1 secretability and ZIKV infectivity in mosquitoes. a-c, NS1 secretability in ZIKV-infected Vero cells. We mutated the single amino acid from alanine to valine at residue 188 in the NS1 protein in an infectious clone of ZIKV Cambodian FSS13025 strain (FSS13025-A188V strain). The FSS13025 or FSS13025-A188V strain (0.01 MOI) was used to infect Vero cells. Supernatants from the infected Vero cells were collected from 0 to 5 days after infection. a, The amount of ZIKV NS1 protein was determined by ELISA. b. The ZIKV titre was determined by plaque assay. c, NS1 quantity normalized against average viral titre (ng per p.f.u.). The data were pooled from three independent biological replicates. d, e, NS1 secretability determined ZIKV infectivity in mosquitoes. The b

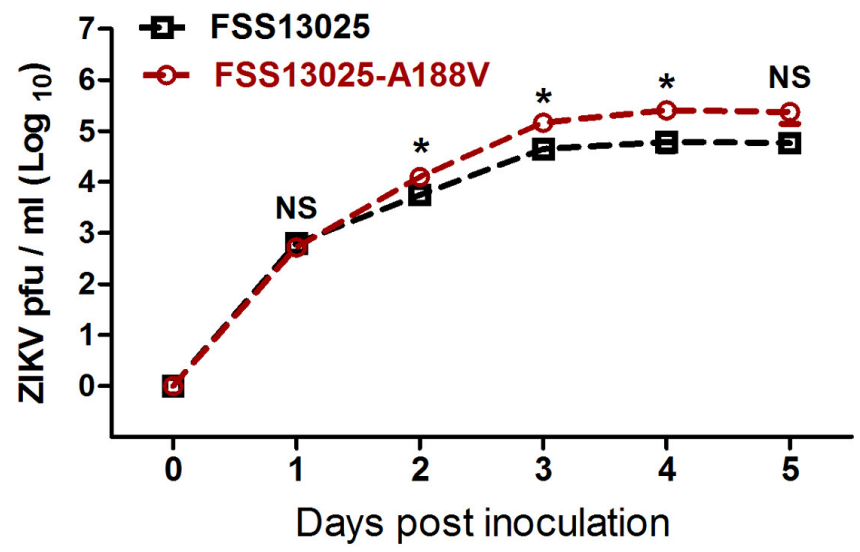

d
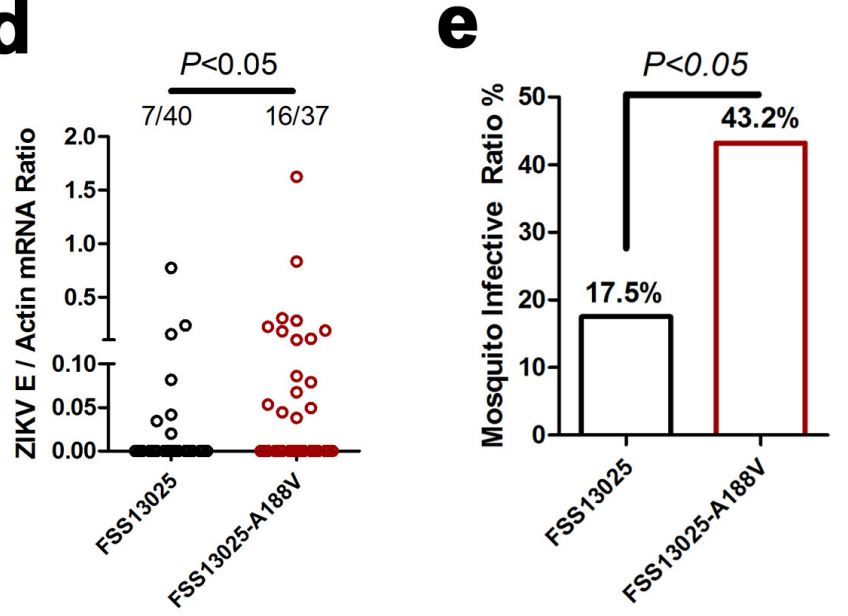

supernatant, isolated from FSS13025-infected or FSS13025-A188Vinfected Vero cells, was premixed with fresh human blood at a 1:1 ratio for an in vitro membrane feeding experiment (final titre $5 \times 10^{4}$ p.f.u. $\mathrm{ml}^{-1}$ ). The fed mosquitoes were killed 8 days after a blood meal to determine the ZIKV load by qPCR. The number of infected mosquitoes relative to the total number of mosquitoes (infected number/total number) is shown at the top of each column. Each dot represents a mosquito (d). The data are represented as the percentage of mosquito infections (e) The data were pooled from three independent biological replicates. Data are mean \pm s.e.m. $(\mathbf{a}-\mathbf{c})$. $P$ values were determined by two-tailed MannWhitney test (a-d) or two-sided Fisher's exact test (e). $* P<0.05$, $* * P<0.01, \mathrm{NS}$, not significant. 
a

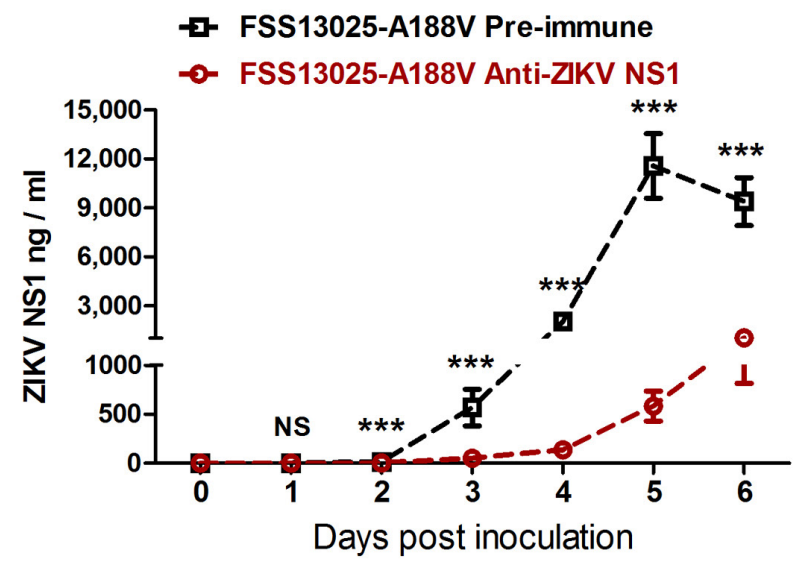

b

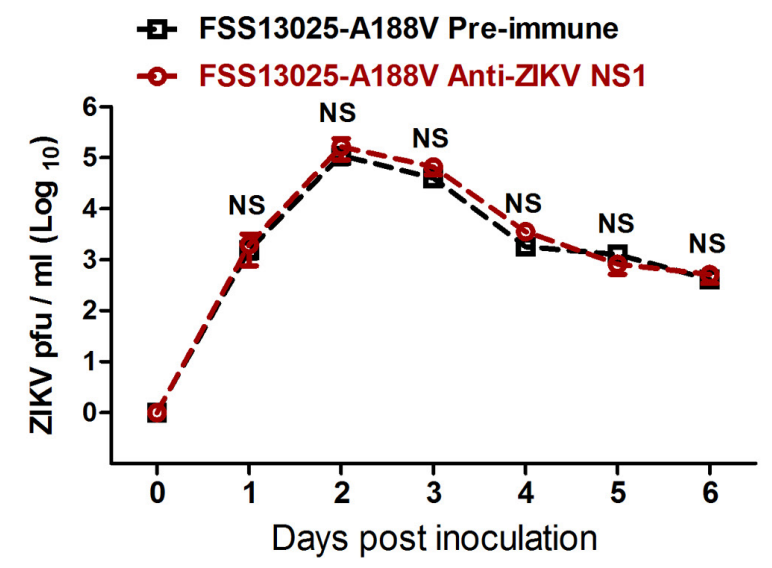

C

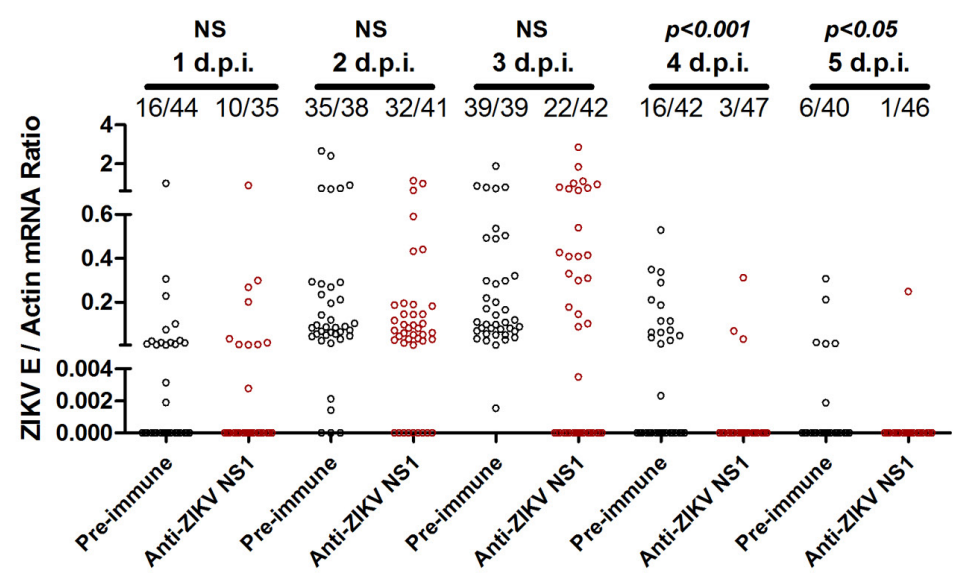

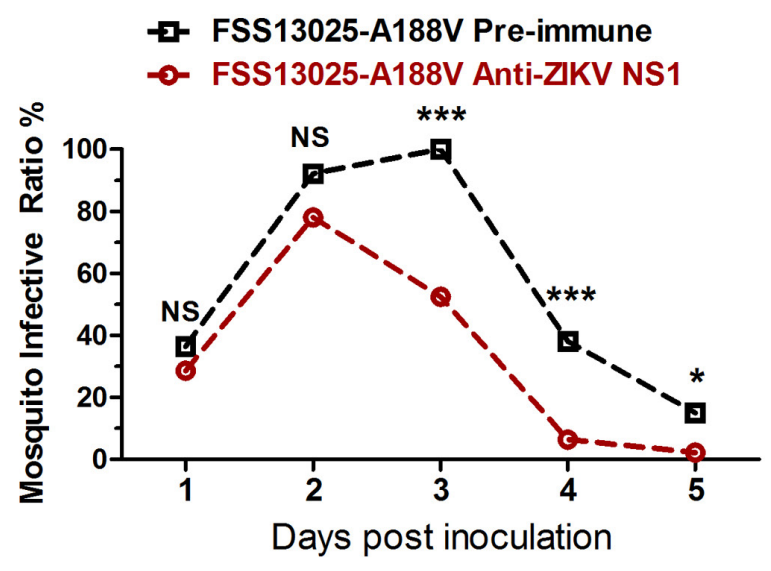

Extended Data Figure 8 | Neutralization of NS1 by mouse ZIKV NS1 antiserum effectively reduced the prevalence of FSS13025-A188V ZIKV in A. aegypti. a, b, AG6 mice were intradermally inoculated with viruses $\left(1 \times 10^{4}\right.$ p.f.u. $)$. ZIKV NS1 was measured using ELISA. Mouse serum was collected to quantify ZIKV NS1 protein from days 0 to 6 after mouse infection (a). The viral load of infectious ZIKV particles in the blood plasma of infected AG6 mice was determined using plaque assay (b). $n=10$ mice per group pooled from 4 independent biological replicates. c, d, Immunoblockade of NS1 in FSS13025-A188V ZIKV-infected AG6 mice reduced the infection of fed A. aegypti ( $n=7$ mice per group pooled from 3 independent biological replicates). The number of infected mosquitoes relative to the total number of mosquitoes is shown at the top of each column. Each dot represents a mosquito (c). The data are represented as the percentage of mosquito infection (d). Data are mean \pm s.e.m. (a, b). $P$ values were determined by two-tailed MannWhitney test $(\mathbf{a}-\mathbf{c})$ or two-sided Fisher's exact test $(\mathbf{d}) . * P<0.05$, $* * * P<0.001$, NS, not significant. 
a

\section{The ZIKV African Lineage}

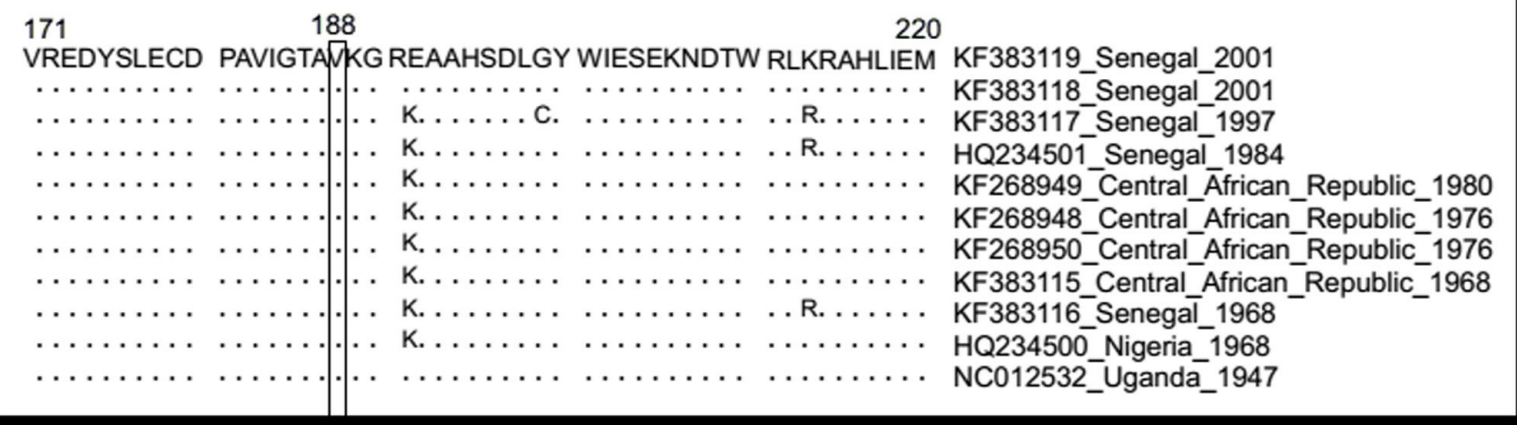

b

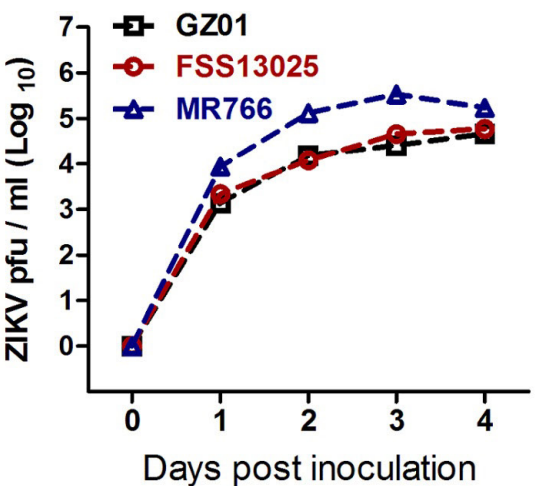

C

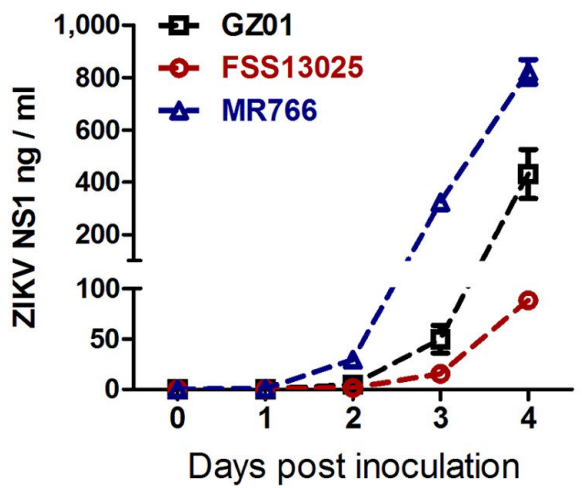

d

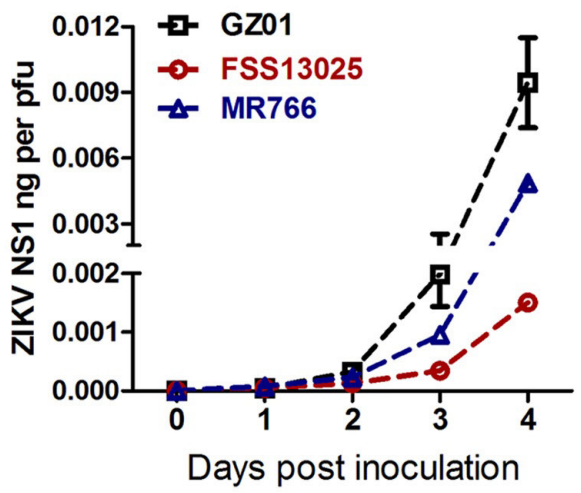

Extended Data Figure 9 | Sequence alignment of NS1 sequences in African ZIKV isolates, and NS1 secretability in African ZIKV-infected Vero cells. a, Sequence alignment of NS1 sequences in African ZIKV isolates. b-d, NS1 secretability in African ZIKV-infected Vero cells. Vero cells were infected at an MOI of 0.01 with the GZ01, FSS13025 and MR766 strains. Supernatants from the infected Vero cells were collected from
0 to 4 days post-infection. $\mathbf{b}$, ZIKV titre was determined by plaque assay. c, The amount of ZIKV NS1 protein was determined using ELISA. d, NS1 quantity normalized against average viral titre (ng per p.f.u.). The data were pooled from three independent biological replicates. The values in the graph represent the mean \pm s.e.m. (b-d). 
Extended Data Table 1 | Primers and probes for gene cloning and qPCR

\begin{tabular}{|c|c|c|c|}
\hline Primers for cloning into pET28a(+) & Upper primer & Lower primer & \\
\hline ZIKV NSI (His tag in both $\mathrm{N}$ and C-terminal) & TATCTAGCTAGCGATGTGGGGTGCTCG & GATTCCGCTCGAGTGCAGTCACCATTGA & \\
\hline Primers for cloning into $\mathrm{pMT} / \mathrm{BiP} / \mathrm{N}$-His/A & Upper primer & Lower primer & \\
\hline ZIKVNSI (VS tag in C-terminal) & TCTCGGGGTACCTGATGTGGGGTGCTCG & TATTACGCTCGAGTGCAGTCACCATTGAC & \\
\hline Primers for cloning into $\mathrm{pcDNA} 3.1 / \mathrm{N} 5$-His/TOPO & Upper primer & Lower primer & \\
\hline Zika prM-E-NS1 (V5 Tag in C-terminal) & TAAGGCGGTACCGCCACCATGCAAAAAGTCATATAC & TATCCGCTCGAGCGTGCAGTCACCATTGA & \\
\hline FSS13025 V-M mutation ( $E$ ) & ATGTGGTTGGGTCTGAATACAAAGAATGGA & TCCATTCTTTGTATTCAGACCCAACCACAT & \\
\hline FSS13025 A-V mutation (NS1) & ATTGGAACAGCCGCTAAGGGAAAGGAGGCT & AGCCTCCTTTCCCTTAGCGGCTGTTCCAAT & \\
\hline P6-740 A-V mutation (NS1) & GTCATAGGAACAGCTGTTAAGGGAAAGGAG & CTCCTTTCCCTTAACAGCTGTTCCTATGAC & \\
\hline The primers for Taqman RT-qPCR & Upper primer & Lower primer & Probe (for Taqman QPCR) \\
\hline ZIKV envelope gene & CCGCTGCCCAACACAAG & CCACTAACGTTCTTTTGCAGACAT & FAM-AGCCTACCTTGACAAGCARTCAGACACTCAA-TAMRA \\
\hline Aedes aegppti actin & GAACACCCAGTCCTGCTGACA & TGCGTCATCTTCTCACGGTTAG & FAM-AGGCCCCGCTCAACCCGAAG-TAMRA \\
\hline Mouse actin & AGCCATGTACGTAGCCATCCA & TCTCCGGAGTCCATCACAATG & FAM-TGTCCCTGTATGCCTCTGGTCGTACCAC-TAMRA \\
\hline
\end{tabular}

